\title{
How to make big molecules fly out of liquid water: applications, features and physics of laser assisted liquid phase dispersion mass spectrometry $\dagger \ddagger$
}

\author{
Ales Charvat ${ }^{a}$ and Bernd Abel ${ }^{* a b}$ \\ Received 17th October 2006, Accepted 5th March 2007 \\ First published as an Advance Article on the web 27th March 2007 \\ DOI: $10.1039 / \mathbf{b 6 1 5 1 1 4 k}$
}

\begin{abstract}
Applications, features, and mechanistic details of laser assisted liquid phase dispersion mass spectrometry are highlighted and discussed. It has been used in the past to directly isolate charged molecular aggregates from the liquid phase and to determine their molecular weight employing sensitive time-of-flight mass spectrometry. The liquid matrix in this MALDI (matrix assisted laser desorption and ionization) type approach consists of a $10 \mu \mathrm{m}$ diameter free liquid filament in vacuum (or a free droplet) which is excited with a focused infrared laser pulse tuned to match the absorption frequency of the $\mathrm{OH}$-stretch vibration of bulk water near $2.8 \mu \mathrm{m}$. Due to these features we will refer to the approach as free liquid matrix assisted laser dispersion of ions or ionic aggregates (IR-FL-MALDI), although also LILBID ("laser induced liquid beam (bead) desorption and ionization") has been proposed early as a descriptive acronym for the technique and may be used alternatively. Low-charge-state macromolecular adducts are isolated in the gas phase from solution via a yet poorly characterized mechanism which sensitively depends upon the laser intensity and wavelength, and after the gentle liquid-to-vacuum transfer the aggregates are analyzed via time-of-flight (TOF) mass spectrometry (MS). Possible mechanisms for the isolation and charging of biomolecules directly from liquid solution are discussed in the present contribution. Recent technical advances such as minimizing the sample consumption, strategies for high throughput mass spectrometry, and coupling of liquid beam MS with HPLC will be highlighted as well. An interesting feature of IR-FL-MALDI is what we call the linear response, i.e., a surprising linearity of the gas phase mass signal on the solution concentration over many orders of magnitude for a large number of biomolecular systems as well as ions. Due to these features the approach may be regarded as a true solution probing spectroscopy, which enables elegant biokinetic studies. Several experiments in which time resolved IR-FL-MALDI-MS has recently been employed successfully are given. A particular highlight is the possibility to quantitatively detect oxidation states in solution, which clearly distinguishes the present approach from other established MS source concepts. Due to the good matrix tolerance also proteins in complex mixtures can be monitored quantitatively.
\end{abstract}

\section{Introduction}

In the past, mass spectrometry was confined to the realm of small molecules: large molecules did not survive desorption and ionization without fragmentation. ${ }^{1}$ The development of new "mild" desorption and ionization methods has revolutionized the analysis of large biomolecules, making mass spectrometry an important tool for biological research. ${ }^{2}$ During the past decade mass spectrometry has seen increasing applications in both chemical ${ }^{3}$ and biological sciences. ${ }^{4}$ The possibility of transferring large charged biomolecules into the gas

\footnotetext{
${ }^{a}$ Max-Planck Institut für biophysikalische Chemie, Am Fassberg 11, 37077 Göttingen, Germany

${ }^{b}$ Institut für Physikalische Chemie der Universität Göttingen, Tammannstrasse 6, 37077 Göttingen, Germany. E-mail: babel@gwdg.de

$\dagger$ This work was presented at the 7th Workshop on Spectroscopy and Dynamics of Molecular Coils and Aggregates, March 8-10, 2006, Göttingen.

$\ddagger$ The HTML version of this article has been enhanced with colour images.
}

phase without significant fragmentation employing MALDI ${ }^{5,6}$ or electrospray ionization $(\mathrm{ESI})^{7}$ has started and fueled important new fields such as proteomics. ${ }^{8,9}$ In fact, the potential applications in biology have been a strong motivation behind recent advantages in this technology. Although a significant number of variants of these techniques (such as laser desorption off silicon surfaces ${ }^{10}$ ), desorption electrospray ionization, ${ }^{11}$ and alternative ionization techniques (e.g., fast atom bombardment, laser spray and sonic spray ionization $)^{1}$ have been developed, ESI and MALDI dominate the field in commercial mass spectrometers.

IR-FL-MALDI in vacuum combined with mass spectrometry is an intriguing technique to isolate charged molecular aggregates of large (bio)polymers directly from the liquid phase and to analyze them employing a mass detector. First attempts to use $\mathrm{CO}_{2}$-lasers for the evaporation of liquid filaments in vacuum for liquid chromatography coupling were reported by Vestal et al. as early as $1978 .{ }^{12}$ IR-MALDI $^{13,14}$ in particular with desorption of biomolecules in ice matrices ${ }^{15}$ has been proposed and established during the first half of the 
past decade by the Hillenkamp group. Brutschy's group has first used Faubel's liquid microfilament ${ }^{16-18}$ in vacuum and a $\mathrm{CO}_{2}$-laser to isolate charged biomolecules directly from the liquid phase and detect them in the gas phase via mass spectrometry without post ionization (LILBID, laser induced liquid beam ionization/desorption). ${ }^{19}$ Later, the same group succeeded in employing a free water filament in vacuum and the desorption of charged biomolecules directly from liquid water with an optical parametric oscillator (OPO). ${ }^{20}$ Some time ago Abel and Faubel also established the IR-FL-MALDI technique in Göttingen. ${ }^{21}$ Just recently Morgner et al. employed LILBID with free liquid water droplets. ${ }^{22}$ In the past decade B. Brutschy's group in Frankfurt ${ }^{20,22-30}$ and the Abel group in Göttingen ${ }^{21,31-37}$ have reported a number of applications of this technique.

Mass spectrometry of organic molecules deliberated from the surface of liquid beams in vacuum with lasers has been an active field of research in the past. ${ }^{38}$ In a series of studies ultraviolet (UV) and alternatively IR laser desorption, often in combination with post-ionization with a second laser, were employed. ${ }^{38-48}$ Although few groups have succeeded in the IR laser desorption and post-photoionization of organic and small biomolecules, ${ }^{49,50}$ only two groups have succeeded in the laser induced soft "desorption" and "ionization" (i.e., isolation of preformed ionic aggregates) of supramolecular systems from liquid microbeams or droplets in vacuum with one IR-laser. In this contribution we will focus on the laser induced isolation of (charged) biomolecules directly from the liquid phase in vacuum with a single IR laser only and their subsequent mass analysis. In this context also a discussion of atmospheric (ap) IR-MALDI and ap-continuous flow MALDI related to the technique described here (in particular if liquid matrices are employed $)^{51}$ appears tempting but in order to keep the focus narrow it will be beyond the scope of the present article.

Although a number of articles have demonstrated the power of the technique, the mechanism(s) of the biomolecule "desorption" is discussed controversially. With regard to "the mechanism" of the process of ion isolation from liquid water, we will first summarize early models that have been proposed to account for some features of IR-FL-MALDI and, in turn, develop a more general mechanistic picture that is supported by our own experimental results and to some extent by theory, being aware of the fact that the mechanism(s) may vary and depend strongly upon the experimental conditions.

The potential of mass spectrometry (MS) for the analysis of body fluids or the diagnosis of diseases is intriguing and forms the basis of clinical essays. ${ }^{9,52}$ Given the complexity of body fluids in terms of species present in solution, the use of MS appears straightforward and logical. ${ }^{53}$ Scientists have dreamed about unlimited species recognition with high-resolution mass spectrometers without the need for cumbersome separation steps and easy quantification of species. Unfortunately, mass resolution and the multiplex advantage is not always the point. Often matrix effects and quantification are a major challenge even with modern mass spectrometers equipped with powerful MALDI and ESI devices. It is probably fair to state that many problems stem from the ionization or desorption step already and are therefore difficult to compensate with a powerful MS downstream. Therefore, we feel that there is a large demand for novel ionization or desorption techniques with improved matrix tolerances and better species quantification, as well as high throughput options beyond the two established techniques.

In previous articles on IR-FL-MALDI-MS the power of the approach was mostly demonstrated for the study of biomolecules at equilibrium. ${ }^{19,31}$ However, the power of the technique, in particular in the continuous mode, appears to be even superior and well suited for time-resolved kinetic studies of biomolecules. It has been demonstrated recently that the added challenge of time scale is not a problem and that educts, products as well as transient intermediates can be monitored even on time-scales that vary over many orders of magnitude. $^{32}$

This article presents some snapshots of the state-of-the-art and recent developments of IR-FL-MALDI (or LILBID) mass spectrometry with the goal of isolating preformed ions or ionic aggregates directly from the liquid phase in vacuum employing IR laser excitation of the liquid matrix. In addition, an overview of characteristic results, generated with this technology will be given. The article may also provide some route maps and ideas for further developments in the near future. While acknowledging the great debt owed to early pioneers and present travellers along the way, the snapshots will be taken largely through the authors' own, admittedly narrow aperture lens, implying that the examples chosen are often from the authors lab. This feature article is not aimed at a complete summary of all IR-FL-MALDI results of the still small but steadily increasing community, but at highlighting characteristic applications, differences to other approaches, as well as domains in which this technique has already proven to be powerful and valuable and where it may become a complement to existing MS-techniques like ESI or MALDI.

The present article is organized as follows: after a general introduction in chapter 1 the overall and specific features and more technical issues of the IR-FL-MALDI approach are summarized and discussed in chapter 2. After having set the stage for the technique some characteristic applications are selected and highlighted in chapter 3 . The chapter 4 concludes the featuring article and gives an outlook onto the promising road ahead with this approach.

\section{Concepts and features of free-liquid matrix assisted infrared laser dispersion of ions and ionic aggregates (IR-FL-MALDI)}

\subsection{Aqueous and volatile liquids in vacuum}

In the language of MALDI, the matrix in IR-FL-MALDI-MS may be a highly volatile micrometer size liquid filament in vacuum (see Fig. 1). Unfortunately, liquid water and vacuum are not very compatible. Early attempts to handle water in vacuum failed until the Faubel group managed to generate and control a fast flowing liquid beam for photoelectron spectroscopy. ${ }^{16-18,54}$ In a typical liquid beam desorption mass spectrometry experiment a liquid jet with a diameter of about 


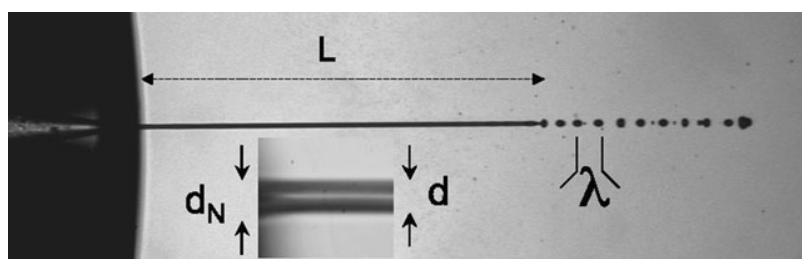

Fig. 1 (a) Circular liquid jets decay into droplets in a distance $L$ from the nozzle. In a limited laminar flow regime the decay is driven by capillary forces leading to spontaneous amplification of surface waves at a critical wavelength $\lambda$, where the beam breaks up into droplets. Contraction of the liquid micro-beam close to the nozzle orifice is visible in the inset, as measured via laser shadowgraphy through a microscope objective. In the figure the back area is the polished surface of the nozzle and the converging channel inside the transparent quartz nozzle is visible.

$10 \mu \mathrm{m}$ emerging from a convergent circular nozzle is used and ejected under high pressure (10-20 bar) into vacuum. It consists of a smooth continuous part of liquid water of a few millimeters, which breaks up into droplets behind a Rayleigh length $L$ (see Fig. 1). The shape of the liquid jet is expected to be influenced by the disturbances first described by Lord Rayleigh. The Rayleigh instabilities are due to sound waves produced by the interaction of the emerging jet with the orifice walls and lead to a breakup of the liquid jet into droplets which are separated by a distance $\lambda$ (Fig. 1). ${ }^{31}$ For a $5 \mu \mathrm{m}$ nozzle, at a typical flow rate of $45 \mathrm{~m} \mathrm{~s}^{-1}$, this corresponds to a droplet frequency of $v \leq 2.9 \mathrm{MHz}$.

Near the liquid water surface the liquid microjet has an atmosphere, i.e., a seam of gas with rapidly decreasing density as a function of radial distance. The mean free path in the vapor around the liquid is given by

$$
\lambda[\mathrm{cm}]=\frac{c}{P_{v}[\text { Torr }]}
$$

For water at the freezing point $\left(273 \mathrm{~K}, p_{v}=4.58\right.$ Torr, $c=$ $5.3 \times 10^{-3}$ Torr $\mathrm{cm}$ ) this results in $\lambda=11.5 \mu \mathrm{m}$. As has been shown by Faubel for a liquid jet with a diameter $d$ smaller than $11.5 \mu \mathrm{m}$ the chance of collisions with molecules in the vapor for particles leaving (or entering) the surface will be less than 1 . A theoretical treatment of this aspect is given in ref. 17. Several problems may be encountered in an operation of the liquid microjet. The ideal, non-viscous flow velocity for a thin wall nozzle is related to the nozzle backing pressure $p_{0}$ and the liquid density $\rho$ by the Bernoulli equation $v=\left(2 p_{0} / \rho\right)^{1 / 2}$. To obtain a stable laminar flow it is necessary to operate in a medium range of Reynolds numbers $\operatorname{Re}(250<\operatorname{Re}<1200)$. This corresponds to liquid jet beam velocities of $20-40 \mathrm{~m} \mathrm{~s}^{-1}$ and backing pressures of 10-30 bar. At smaller Re the jet is unstable and forms droplets, while at larger Re the jet starts spraying.

Near the nozzle exit the beam contracts somewhat (due to hydrodynamic focusing and evaporative cooling) within a distance of a few nozzle distances (Fig. 1, inset). In vacuum the liquid micro-jets are expected to rapidly cool by evaporation. The evaporating molecules transport the heat of evaporation away from the liquid core, resulting in cooling of the jet. As the enthalpy of the core is proportional to the temperature, the energy balance is given by

$$
\mathrm{d} T_{0}=\alpha \frac{H}{c_{p}},
$$

where the $\mathrm{d} T_{0}$ is an infinitesimal temperature decrease, $H$ is the heat of vaporization, $c_{p}$ the specific heat capacity of the liquid and $\alpha$ is the ratio of the number of molecules which have evaporated to those remaining in the jet. For a cylindrical jet $\alpha$ is simply the ratio of volumes of an infinitesimal ring of width $\mathrm{d} r_{0}$ and a cylinder, both with lengths $\mathrm{d} z$ :

$$
\alpha=\frac{2 \pi r_{0} \mathrm{~d} r_{0} \mathrm{~d} z}{\pi r_{0}^{2} \mathrm{~d} z}=2 \frac{\mathrm{d} r_{0}}{r_{0}} .
$$

From eqns (3) and (2) the temperature gradient is obtained.

$$
\frac{\mathrm{d} T_{0}}{\mathrm{~d} r}=-\frac{2}{r} \frac{H}{c_{p}} \quad \text { or } \quad \dot{T}_{0}=-2 \frac{\dot{r}_{0}}{r_{0}} \frac{H}{c_{p}}
$$

The time dependence can be transformed in a spatial variation along the $z$-axis by dividing through the velocity of the liquid jet $\mathrm{d} z / \mathrm{d} t=v_{\text {jet }}$.

$$
\frac{\mathrm{d} T_{0}}{\mathrm{~d} z}=-2 \frac{\dot{r}_{0}}{v_{\text {jet }}} \frac{H}{c_{p}} \frac{1}{r_{0}}
$$

$v_{\text {jet }}, c_{p}=4.2 \mathrm{~J} \mathrm{~g}^{-1} \mathrm{~K}^{-1}$, and $H=2.27 \mathrm{~kJ} \mathrm{~g}^{-1}$ are assumed (in a first approximation) to be temperature independent. In contrast, the rate of decrease of the jet radius $\mathrm{d} r_{0} / \mathrm{d} t$ depends on the vapor pressure and thus on the surface temperature of the jet,

$$
\dot{r}\left(T_{0}\right)=\tilde{u}\left(T_{0}\right) \frac{\rho_{\text {vapor }}\left(T_{0}\right)}{\rho_{\text {liquid }}},
$$

where $\tilde{u}\left(T_{0}\right)$ is the mean velocity of the evaporating molecules, which is given for a Maxwellian gas by $\tilde{u}\left(T_{0}\right)=(8 \mathrm{kT} / \pi \mathrm{m})^{1 / 2}$. The vapor density dependence on the liquid temperature $T_{0}$ can be calculated from the Clausius-Clapeyron relation $\rho_{\text {vapor }}=C \exp \left(-\mathrm{H} / k T_{0}\right)$. For a temperature $T_{\text {ref }}=277 \mathrm{~K}$, with $\rho_{\text {liquid }}\left(T_{\text {ref }}\right)=1 \mathrm{~g} \mathrm{~cm}^{-3}$, and $\rho_{\text {vapor }}\left(T_{\text {ref }}\right)=6.4 \times 10^{-6} \mathrm{~g}$ $\mathrm{cm}^{-3}$, this leads to the reduced expression,

$$
\dot{r}_{0}\left(T_{0}\right)=\dot{r}_{\text {ref }}\left(\frac{T_{0}}{T_{\text {ref }}}\right)^{1 / 2} \exp \left[-\frac{H}{k T_{\text {ref }}}\left(\frac{T_{\text {ref }}-T_{0}}{T_{0}}\right)\right]
$$

The above constants for $\mathrm{H}_{2} \mathrm{O}$ yield for the rate of evaporative ablation $\mathrm{d} r_{\text {ref }} / \mathrm{d} t=0.36 \mathrm{~cm} \mathrm{~s}^{-1}$ at $T_{\text {ref }}=277 \mathrm{~K}$. A differential equation for the change of $T_{0}$ with $z$ can be obtained which can be integrated numerically. Calculated temperatures in comparison with experimental values are given in ref. 17. It should be noted that evaporative cooling of liquid micro-jets may be used to study super-cooled liquid water down to less than $210 \mathrm{~K}$. The unavoidable phase transition happens in our vacuum setup after a free flight of the droplets in vacuum of $50-100 \mathrm{~cm}$, which then arrive as ice particles in the liquid nitrogen cold trap. Using the technology described above liquid jets with diameters down to 2.5 micrometer diameter have been generated in the lab of the authors. ${ }^{34}$ The every day working nozzle diameter is, however, $10 \mu \mathrm{m}$ resulting in a liquid beam of $7 \mu \mathrm{m}$. The liquid beam temperature in the working range at distance $x=1-2 \mathrm{~mm}$ from the nozzle orifice is usually close to the freezing point of water. 

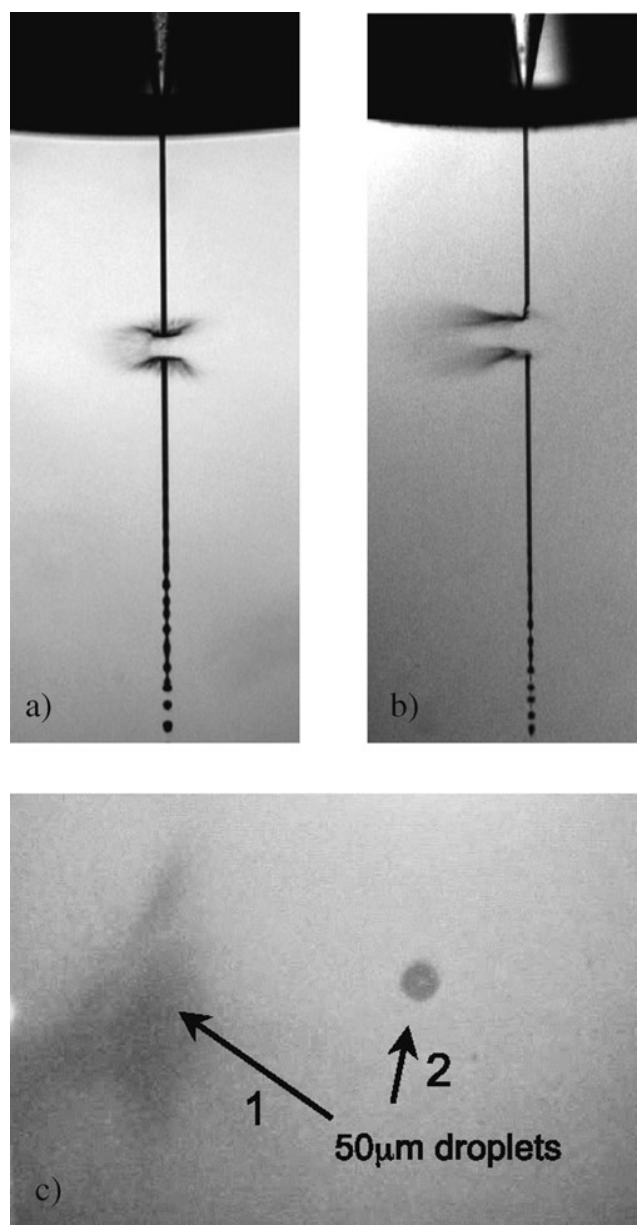

Fig. 2 Dispersion/desorption from a $10 \mu \mathrm{m}$ liquid water beam with an IR laser. (a) Large penetration depth $(\lambda=2.680 \mu \mathrm{m})$. (b) Small penetration depth $(\lambda=2.810 \mu \mathrm{m})$. (c) Dispersion/desorption from small $50 \mu \mathrm{m}$ water droplets with an IR laser $(\lambda=2.810 \mu \mathrm{m})$. Droplet 1 is dispersed and droplet 2 serves as a reference.

A free liquid matrix in vacuum (to be used in IR-FLMALDI-MS) may also be a single droplet or aerosol droplets generated with a dedicated droplet source (see Fig. 2). The problem in both cases-liquid nano-droplet aerosols or micrometer sized liquid droplets on demand require the generation of droplets in a region of relatively high pressure, otherwise the droplet generators (either piezo driven capillaries or single droplet generators such as the MICRODROP ${ }^{\mathrm{TM}}$-generator) do not work in vacuum and ultimately freeze due to strong evaporative cooling. This obstacle requires the generation of droplets at relatively high pressures (near or somewhat below $1 \mathrm{~atm}$ ) and a suitable and well-pumped high-pressure-vacuum interface and powerful differential pumping. For aerosol and droplet on demand mass spectrometry a differential pumping stage with a set of aerodynamic lenses transfer and collimate the droplets into high vacuum via hydrodynamic focusing. ${ }^{55}$ Then the droplets are detected optically and excited with the desorption laser (Fig. 2c). ${ }^{56}$ For general or more specific information about the generation of droplets and the transfer into the vacuum system we refer to ref. $22,55,57-59$.

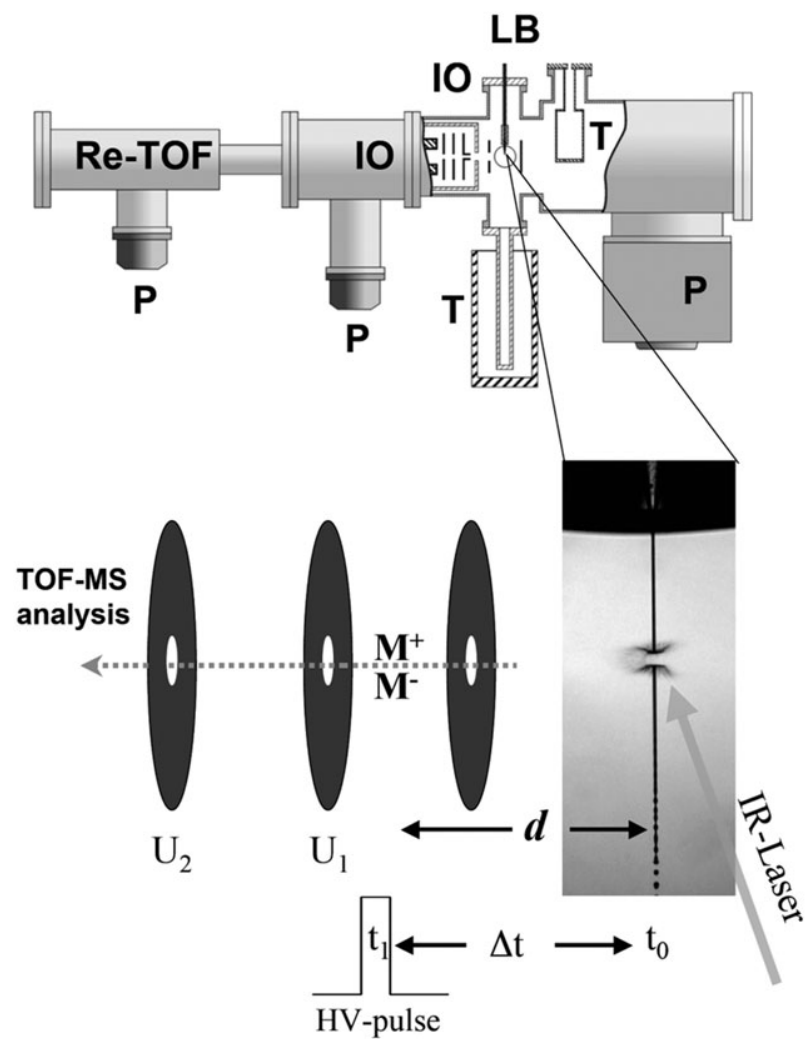

Fig. 3 Experimental setup and desorption scheme. Re-TOF: reflectron time-of-flight mass spectrometer; P: pumps; T: liquid nitrogen cryo trap and cryo trap for the liquid beam; LB: nozzle with $x, y$, $z$-manipulator; IO: differential pumping stage including the ion optics; $U_{1}, U_{2}$ : accelerating voltages of the ion optics; $d$ : distance between liquid beam and ion gate; $\Delta t$ : time between desorption and trigger of the ion optics. The length of the HV-trigger pulse is $t_{1} \sim 2 \mu \mathrm{s}$. The time resolution of the high speed stroboscopy picture of the exploding micro-beam is around $10 \mathrm{~ns}$. For more details see the text.

\subsection{IR-FL-MALDI employing liquid (water) micro-beams}

A detailed description of an experimental apparatus employing liquid micro-beams in combination with mass spectrometry is given in ref. 28,31 and 34 . For experiments like those presented mainly in this feature article we employ a $10 \mu \mathrm{m}$ thin water beam with a flow speed of $\sim 40 \mathrm{~ms}^{-1}$ containing the molecules under investigation (Fig. 2a, b). It is ejected through a pulled quartz nozzle (lifetime of a nozzle is between 3-6 months) with high pressure (10-20 bar) into the vacuum chamber (see Fig. 3) employing a standard HPLC pump (GyNKoteK). Clogging is efficiently suppressed with a filter in the high-pressure line. The liquid micro-beam is dumped in a cryo-trap whose content has to be disposed of, usually after about $10 \mathrm{~h}$ of operation by venting the apparatus. The temperature of the beam can be controlled precisely with a thermostat and a cooling/heating jacket around the nozzle mount. IR-FL-MALDI works also with other solvents (alcohols) and solvent mixtures (acetonitrile, alcohols, etc.) as long as the water (or alcohol) content is above $20-30 \%$. Tunable nanosecond infrared radiation at a repetition rate of $20 \mathrm{~Hz}$ and a pulse energy between $0.5-2 \mathrm{~mJ}$ was generated with an optical parametric oscillator (GWU). The wavelength was 
tuned at the blue side of the $\mathrm{OH}$ bulk absorption band of water in the range $2600-2850 \mathrm{~nm}$. The IR beam was expanded and focused carefully onto the liquid beam reaching an intensity of less than $10^{8} \mathrm{~W} \mathrm{~cm}^{-2}$ incident on the beam (see Fig. 2). The continuous part of the liquid jet is irradiated in the focus of a $7 \mathrm{~ns}$ long IR laser pulse tuned nearly in resonance with the $\mathrm{OH}$-stretch absorption of bulk water. Depending upon the wavelength the penetration depth of the IR radiation is between 1 and $10 \mu \mathrm{m}$ (Fig. 2). The absorbed energy of the IR laser in water is redistributed within picoseconds ${ }^{60}$ and, in turn, heating and dispersing the irradiated volume. A limited fraction of the isolated charged (water) aggregates moves in field-free space to the entrance aperture of a skimmer and enter the ion optics of the mass spectrometer. During this transit time of a few microseconds continuous evaporation of water from the aggregates may dissipate heat and, in turn, stabilize the aggregates and eventually lead to isolated or clustered biomolecules with a low net charge (e.g., attachment of protons).

The time dependent voltages $U_{1}$ and $U_{2}$ in the ion optics in Fig. 3 of the spectrometer first decelerate and then accelerate the molecules for their final mass analysis. In addition, the pulsed ion optics act as an ion gate for the incoming broad velocity, i.e., translational energy distribution of the ionic aggregates, which significantly improves mass resolution. In the experiments the mass spectrometer could be operated in the positive or negative ion mode.

As displayed in Fig. 3 the ion generation in the Göttingen setup was outside the ion optics. The advantage of improved mass resolution and the gained time for desolvation is obvious. However, due to the velocity distribution of the disintegrating beam and a small mass effect due to a velocity slip in the "biomolecule seeded" water gas beam, the delay time (see Fig. 3) has to be scanned or adjusted in order to obtain the whole mass spectrum. The more traditional way of placing the water beam inside the ion optics on the other hand also has significant disadvantages, such as electric discharges, etc. Also in the latter case the explosion can only be controlled via delayed extraction of ions. Also in this configuration a mass effect may be present as a function of the delay time for extraction.

An internal ion gate in the Wiley-McLaren focus of the spectrometer enabled us to further suppress unwanted ions and background. The repeller and extraction electrodes of the mass spectrometer are switched to typically 4-6 kV after a variable delay time, $\Delta t$, allowing the acceleration and subsequent mass analysis of a fraction of the total velocity distribution of the desorbed species (see Fig. 3). In front of the CHEVRON type multi-channel plate detector (not shown) the ions were post-accelerated with $10 \mathrm{kV}$ in order to improve detection efficiency for higher masses. The ion optics as well as the detector were powered by a bipolar power supply. Different ion modes could be selected with an electric switch. Time-of-flight spectra were recorded after amplification with a $150 \mathrm{MHz}$ 8-bit digitizer card (ACQIRIS). The typical mass resolution of the spectrometer operated with liquid beam desorption was around $m / \Delta m=2000-600$ between 1000 and $10000 \mathrm{Da}$. For mass calibration in the low mass region we have used multimers of arginine and in the high mass region protein mixtures as mass standards (insulin, myoglobin, lysozyme).

\subsection{Liquid injections in a water carrier beam-the SLICED concept}

As outlined above continuous liquid beam desorption mass spectrometry has outstanding advantages but at the same time it has at least one severe drawback. In combination with a low repetition laser the duty cycle is rather poor which is correlated with significant and unnecessary analyte consumption. For a continuous micro-beam of $10 \mu \mathrm{m}$ diameter with a typical flow rate of $0.2 \mathrm{ml} \mathrm{min}^{-1}$ an amount of $12 \mathrm{ml}$ within $1 \mathrm{~h}$ is used. At a concentration of $10^{-5} \mathrm{M}$ a sample of $1.2 \times 10^{-7} \mathrm{~mol}$ corresponding to $1.2 \mathrm{mg}$ of a $10000 \mathrm{Da}$ biomolecule is consumed. Even if the time for recording a mass spectrum and performing a mass analysis takes much less than an hour, the point to make here is that the duty cycle for the above approach must be improved, because for many analytical applications such a material consumption is not tolerable.

Recently, we have developed a technique for a local injection of a sample into the main carrier beam ${ }^{32}$ to reduce the sample consumption in this type of experiment following up on an early approach by Sobott et al. ${ }^{28}$ In their work they have used manual HPLC valves to bypass the main water reservoir line for some time in order to reduce the sample consumption, with sample loops down to $100 \mu$ providing sample detection for roughly $5 \mathrm{~min}$ which was sufficient for alignment and recording a mass spectrum. ${ }^{61}$ With modern automatic highpressure valves (HPLC technology) the main water flow cannot only be bypassed for some time but very small samples (100 nl-1 $\mu \mathrm{l})$ can be injected directly, providing transient "plug flows" well below a second. We have termed this approach seeded liquid carrier excitation and desorption (SLICED). ${ }^{32} \mathrm{~A}$ small sample of the analyte solution (e.g. 1-2 $\mu \mathrm{l})$ is injected into the carrier beam before the nozzle. For this approach we use automatic rapidly switching high-pressure valves (RHEODYNE) that are filled at ambient pressure. For laminar flow conditions and small dead volumes as well as thin capillaries the segment flow is not broadened too much and can be read out by the IR desorption laser. In Fig. 4 we have displayed a representative example for a carrier beam flow of $0.5 \mathrm{ml} \mathrm{min}{ }^{-1}$, a sample volume of $2 \mu \mathrm{l}$, and an inner diameter of the capillaries of $130 \mu \mathrm{m}$. The transient event has been monitored with a fluorescent dye (coumarin) excited by an argon ion laser. The time-scale of the transient injection at present enables us to record 10-20 spectra on the transient, which does not require special synchronization. Indeed, about this number of spectra are required to obtain an acceptable signal-to-noise spectrum of cytochrome $\mathrm{c}\left(10^{-5} \mathrm{M}\right)$ also displayed in Fig. 4. With an easy to handle probe volume of $2 \mu \mathrm{l}$ the sample consumption is already on the order of 20 picomol, corresponding to a material consumption that is already orders of magnitude lower than with the continuous technique. At present the automatic injection of volumes on the order of 10-100 nanoliters is under development in our laboratory finally using $1 \mathrm{kHz}$ IR-desorption lasers. With the now available (high speed) HPLC injector valves (Rheodyne) the material consumption for an analysis in this type of 


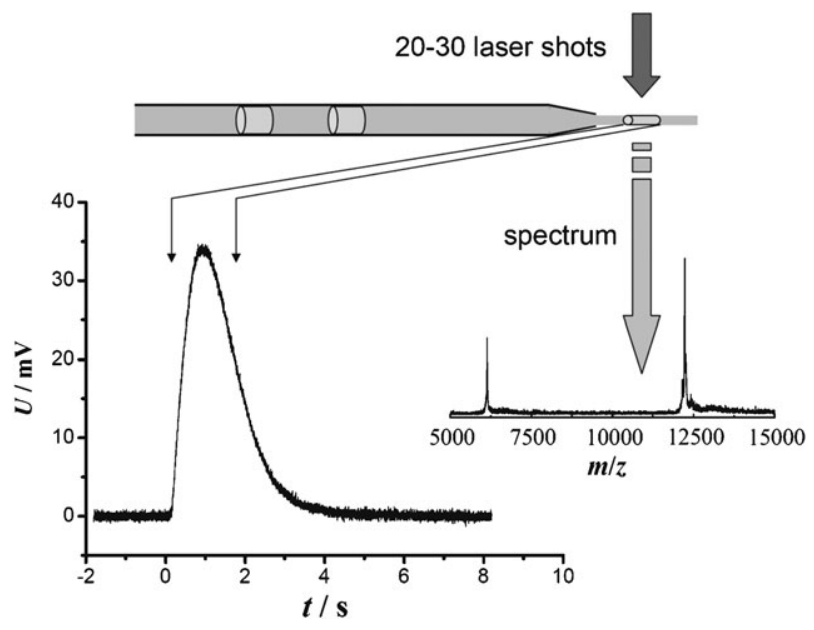

Fig. 4 Laser induced fluorescence of an argon ion laser excited liquid segment (containing a coumarin dye) injected with a high pressure valve demonstrating the SLICED concept (segment not drawn to scale). During the transient (HWFM $=1.5 \mathrm{~s}$ ) a mass spectrum of cytochrome c (pos. ion mode) can be obtained from 20 laser shots. For more details see the text.

approach may be expected in the femtomol region in the near future.

It should be noted at this point that with the SLICED method the sample consumption has been improved significantly as compared with the early LILBID method, however, if only sample consumption is important the IR-FL-MALDI or LILBID approach with single liquid droplets on demand or aerosol MALDI is the method of choice. Droplet on demand technology is particularly nice if the problem of dead volumes is solved, which usually limits the overall and effective sample consumption. In other cases, e.g., in kinetic studies like those presented in this article the classical continuous liquid microbeam approach may be advantageous, because the control of the liquid matrix is more convenient and more flexible. Temperature control, varying temperatures, solvent gradients, $\mathrm{pH}$ control, and kinetic measurements, incorporating fast mixing devices, as well as separation steps (HPCL, chromatography) appear easier with the latter approach. Also, in terms of high throughput sampling and analysis the continuous IRFL-MALDI-MS has some advantages. As displayed in Fig. 4, the time for one single and independent measurement (20 shot averaging) is short and can easily be optimized to be significantly less than a second. A sampling rate of one independent sample per second would correspond to a very high-throughput sampling rate. Even when compared with the highest throughput sampling technique available today, this feature is rather fascinating (although at the price of a not so low material consumption at present). It should be emphasized again that this is the effective (real) analysis time without large preparation and processing the sample (such as necessary with MALDI techniques). If separation steps are necessary, the technique may be coupled with HPLC. In such a case the "seeded water beam" can be fed by several HPLC machines, and the independent analytic samples are "read out" and analyzed by the IR-laser and the mass spectrometer.
2.4 IR-FL-MALDI employing liquid micro-droplets-towards very low sample consumption

As noted earlier, IR-FL-MALDI-MS can also be performed with liquid droplets in vacuum (Fig. 2c). ${ }^{56}$ Here the advantage of low sample consumption is evident. Both, mass spectrometry of aerosol droplets and larger droplets on demand have been reported by a number of groups. ${ }^{22,55,58,59,62-64}$ The typical configuration employs single-laser desorption-ionization to produce a pulse of ions from each selected particle, ${ }^{64}$ nevertheless, also powerful two photon approaches have been reported ${ }^{55,58}$ which, in some cases, even provide the possibility of depth profiling. ${ }^{59}$ Aerosol mass spectrometry has been pioneered by Prathers's group ${ }^{64}$ and Murray and Russel. ${ }^{62}$ While Baer and Miller have used this technology for the analysis of organic molecules in aerosols, ${ }^{55,58,59}$ Frank and co-workers ${ }^{65}$ have even identified whole organisms (bacteria) via laser desorption/ionization aerosol mass spectrometry. It appeared straightforward to develop the LILBID technique into the direction of using single droplets instead of microliquid filaments. Following up on powerful concepts for droplet transfer and handling in vacuum, well established for aerosol mass spectrometry, Morgner et al. have demonstrated that for LILBID (with B as an abbreviation for "bead") with synchronized droplets on demand the material consumption can be dramatically reduced as opposed to a continuous liquid beam and even the SLICED technique. ${ }^{22}$ If a droplet of $50 \mu \mathrm{m}$ has a volume of about $50 \mathrm{pL}$ and if a spectrum could be taken with one droplet only $\left(10^{-6} \mathrm{M}\right)$ the material consumption in ideal cases may approach the low femtomol or even the attomol range. Beyond the fact that spectra usually take more than one droplet, the problem of dead volumes has to be resolved, in order not to be limited by dead volumes rather than by droplet volume. Also the stability of droplets in space and synchronization may induce problems. Nevertheless, efforts to solve these problems are underway in several groups now. Just recently, Brutschy and co-workers have reported first experiments using single $50 \mu$ m-diameter droplets on demand for the analysis of biomolecules and biomolecular complexes with IR laser desorption mass spectrometry. In Fig. 5 the mass spectrum of lysozyme $\left(10^{-5} \mathrm{M}\right)$ is displayed. Also in this case single droplets were generated with a piezo-driven droplet generator (MICRODROP) and transferred into vacuum via a differential pumping stage. ${ }^{22}$ The size of the droplets was chosen as a compromise between small sample consumption (65 pL sample) and stability as well as long-term operation of the system. The mass spectrum in Fig. 5 has been taken from 10 droplets corresponding to a sample consumption of $6 \mathrm{fmol}$.

\subsection{IR-FL-MALDI and classical MALDI}

Classical matrix-assisted laser desorption/ionization (MALDI) consists in mixing the compound to be analyzed in a solvent containing in solution small organic molecules (e.g., DHB, dihydroxybenzoic acid), called a matrix, and having a strong absorption at the desorption laser wavelength. By evaporation of the solvent a solid solution of the sample in the matrix is obtained. The irradiation of this mixture induces the accumulation of a large amount of energy in the condensed phase through electronic excitation of the matrix molecules. 


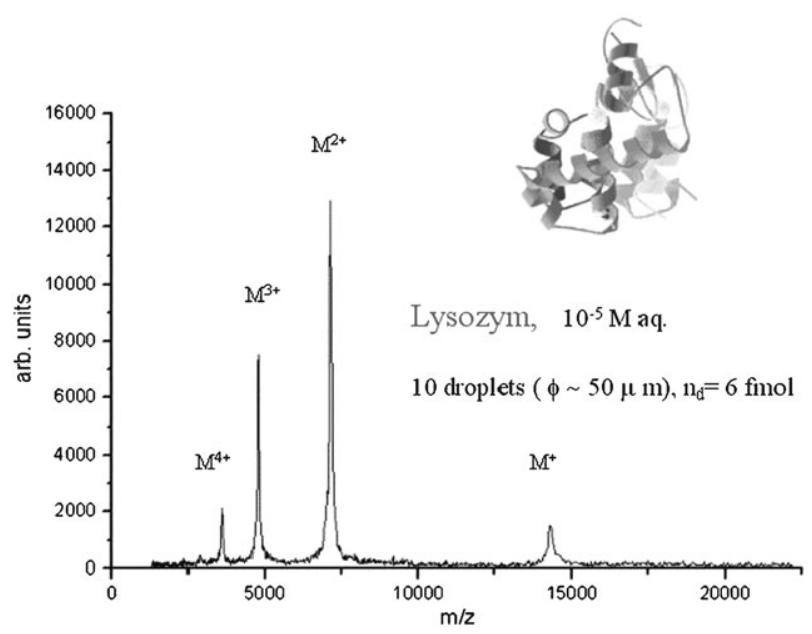

Fig. 5 LILBID spectrum of lysozyme from 10 droplets $(\phi=50 \mu \mathrm{m})$ at a concentration of $10^{-5} \mathrm{M}$ (pos. ion mode). Data and figure (unpublished results) provided by B. Brutschy.

This causes desorption of the ions formed, e.g., by proton transfer in the gas phase between the photoexcited matrix and the analyte compound. Because the desorption conditions are far from thermal equilibrium, the analyte molecules are usually not fragmented to a large extent. The mechanism has been the subject of a long-standing debate over the years. Different scenarios have been proposed for the desorption process and the formation of charged species. ${ }^{66,67}$ At least part of the explanation of the desorption process is that through electronic excitation and fast internal conversion (or excited state dynamics) the energy is rapidly transferred to the lattice of the matrix which undergoes a phase transition and evaporates in turn producing unfragmented analyte molecules in the gas phase. By acid base reactions in the gas phase the analyte molecules are protonated or deprotonated and form mostly singly charged adducts. The presence of free electrons during the absorption and conversion of electronic energy into translational degrees of freedom of the matrix molecules may also produce charged molecules. In general MALDI produces positively or negatively charged but in any case low charge state analyte molecules that can be analyzed in the positive or negative ion mode of the spectrometer. For the production of the charged analyte molecules the 'cluster model' and the 'lucky survivor model' have been proposed. ${ }^{66}$ In the first case large clusters containing the analyte are ejected which, in turn, dissociate (after internal acid base reactions) and finally yield the charged analyte molecule whereas in the latter model the detectable amount of charged analyte molecules corresponds to the tiny fraction of charged analyte molecules that do not recombine in the ejection process. There have also been conjectures and experimental evidence that preformed ions in the matrix may be ejected in the desorption process. ${ }^{66,67}$

Also IR-MALDI has been reported, which is, however, much less used today probably due to the larger prices and the availability of suitable IR lasers. ${ }^{68,69}$ A standard matrix for IR-MALDI is glycerol or ice. The desorption mechanism after IR-excitation is distinctly different from the UV-MALDI case but it also produces analyte molecules in low charge states. Also the penetration and the ablation depths are different. The mechanism has not been understood in detail so far. In comparison with the latter approach IR-FL-MALDI-MS also uses IR laser absorption in a liquid (water) matrix to convert laser light into heat and gas driving the ion isolation process and the liquid to vacuum transfer of charged biomolecules. Also MALDI and IR-FL-MALDI-MS can be operated in the positive or negative ion mode. Although there may be several similarities, nonetheless decisive features of the techniques are different. The main and obvious difference is the control and homogeneity of the liquid matrix (temperature, $\mathrm{pH}$ value, composition, mixing properties), as well as the excitation and desorption process. While in UV-MALDI-MS the samples (analyte and matrix) are ablated and the charging of biomolecules is largely driven by proton (and charge) transfer in the gas phase, ${ }^{66,67}$ in IR-FL-MALDI-MS the liquid matrix is excited and highly dispersed up to the isolated protonated or deprotonated (or ion aggregated) analyte molecules. Charging is driven here by solution acid base reactions with the solvent or through adduct formation. ${ }^{31,32}$ In this process, which may depend upon the wavelength and intensity of the desorption laser, the preformed ions or ionic aggregates/complexes are separated, ejected and isolated (see below). It has been demonstrated in the past that the IR-FL-MALDI-MS signals reflect concentrations of charged species in solution rather than proton affinities in the gas phase.$^{61}$ In addition, the technique displays a surprisingly linear response of the gas phase signal as a function of concentration in the liquid over many orders of magnitude, as will be shown below. ${ }^{31,32}$ This is usually not the case for MALDI-MS. Nevertheless, some similarities of IR-MALDI employing frozen ice or glycerol matrices and IR-FL-MALDI-MS are also obvious and the borderline between the approaches becomes diffuse. ${ }^{14,70}$

\subsection{Negative and positive ion modes and charge states}

As the classical MALDI technique, IR-FL-MALDI under typical experimental conditions produces low charge state analyte molecules in the gas phase after liquid matrix desorption. This is not only a feature of small molecules, which have only few sites for protonation and deprotonation but also for relatively large molecules above $5000 \mathrm{Da}$. These findings are actually somewhat different to typical results from the Brutschy group (see also Fig. 5). ${ }^{19}$ They may be due to different solvents or different experimental conditions. In addition, charge state distributions are shifted towards lower values when buffer or ion concentrations (overall "ion strength") increases. The low charge state ions and ionic aggregates from liquid microfilaments may be detected in the positive and negative ion mode as it is standard for $\mathrm{ESI}^{71}$ and MALDI $^{67}$ Usually, in the positive ion mode protonated biomolecules $\left(\mathrm{MH}^{+}\right)$are detected if no other cations in large concentrations are present in solution $\left(\right.$ e.g., $\left.\mathrm{Na}^{+}\right)$. In the presence of $\mathrm{Na}^{+}$or other ions, $\mathrm{M}: \mathrm{Na}^{+}$aggregates are easily detectable if their concentration is above the $\mathrm{H}_{3} \mathrm{O}^{+}$concentration. If the $\mathrm{pH}$ is decreased, e.g., by adding acid, the detectable "ion yield" in the gas phase increases until saturation due to the increase in the overall ion concentration which tends to decrease the overall ion yield. In general, measurements can be made for concentrations below $0.15 \mathrm{M}$ (although optimal 
conditions start at $0.01 \mathrm{M}$ ). For a $\mathrm{NaCl}$ this value belongs to physiological $\mathrm{NaCl}$ solution. Normally, measurements are made in buffered solution at around $\mathrm{pH}=7$ (if not otherwise noted). IR-FL-MALDI-MS is relatively tolerant against buffer concentrations, however, the upper limit for measurements has been found near $0.2 \mathrm{M}$. In the negative ion mode $[\mathrm{M}+\mathrm{OH}]^{-}$and deprotonated biomolecules $[\mathrm{M}-\mathrm{H}]^{-}$or adducts with anions such as $\mathrm{Cl}^{-}$are detected.

It is often discussed - in particular for ESI-MS - whether mass spectrometric techniques can detect actual charge states in solution, which may depend upon the $\mathrm{pH}$ of the solution and the $\mathrm{pKs}$ of the analyte molecules and, in turn, upon their isoelectric point (IP). As an illustrative example we will consider lysozyme ( $\approx 14500 \mathrm{Da}$, IP at $\mathrm{pH}=10.5-11)$. The protein in solution is only 6-fold charged at $\mathrm{pH}=7 .{ }^{61} \mathrm{With}$ LILBID it is detected with up to 4-5 positive charges depending on the solvent (alcohols, water) with a maximum at around 2 (see also Fig. 5). ${ }^{61}$ Typical results we obtain in the positive ion mode are spectra with a dominating charge state of +1 with some contributions of doubly charged species. The differences in the data sets are attributed to differences in the desorption process, although the overall trend is that low charge states are observed in general. With regard to the appearance of charge states IR-FL-MALDI-MS and MALDI-MS behave similarly. With both approaches lysozyme is best detected in low positive charge states. Sobott et al. have also detected lysozyme in the negative ion mode with charge states peaking at $-1 .{ }^{61}$ Since proteins are often detected in the positive ion mode because they have many amino acid residues that can be protonated, it may be somewhat surprising that lysozyme can also be detected in negative ion mode with somewhat reduced sensitivity. Obviously the charge state distribution of lysozyme in solution ranges from +5 to -4 , with a peak between +3 and $+1 .^{61}$ For ESI the average charge state distribution for positive charge states peaks at significantly higher values between +7 and +10 , on average one charge may be expected per $1000 \mathrm{Da}$. The upper limit for the charge state distribution in ESI experiments is limited by the coulomb explosion limit. It is discussed extensively in the literature whether it is possible to estimate the number of sites that can be protonated in a molecule from the population of the largest charge states and whether the detected ESI charge state distributions reflect charge state distributions of the molecule in solution. In any case IR-FL-MALDI (LILBID) cannot measure actual charge state distributions in solution. The detected charge states are usually biased against low charge states with a dominating charge state at +1 or -1 . Although the experiment cannot measure charge states in solution nor charge state distributions accurately, it senses the charge state distribution in a qualitative fashion. It can actually probe the center of gravity of the charge distribution, i.e., whether mainly positively or negatively charged analyte molecules are abundant. For amino acids $\mathrm{p} K$-values are known and the low charge state distribution of the system can be readily calculated. Fig. 6a displays mass spectra for $0.001 \mathrm{M}$ aspartate solution in the negative and in the positive ion modes (Fig. 6b). The relative signal intensities for the parent mass reflect qualitatively the abundance of different charge states in solution. Other lines in Fig. 6 belong to

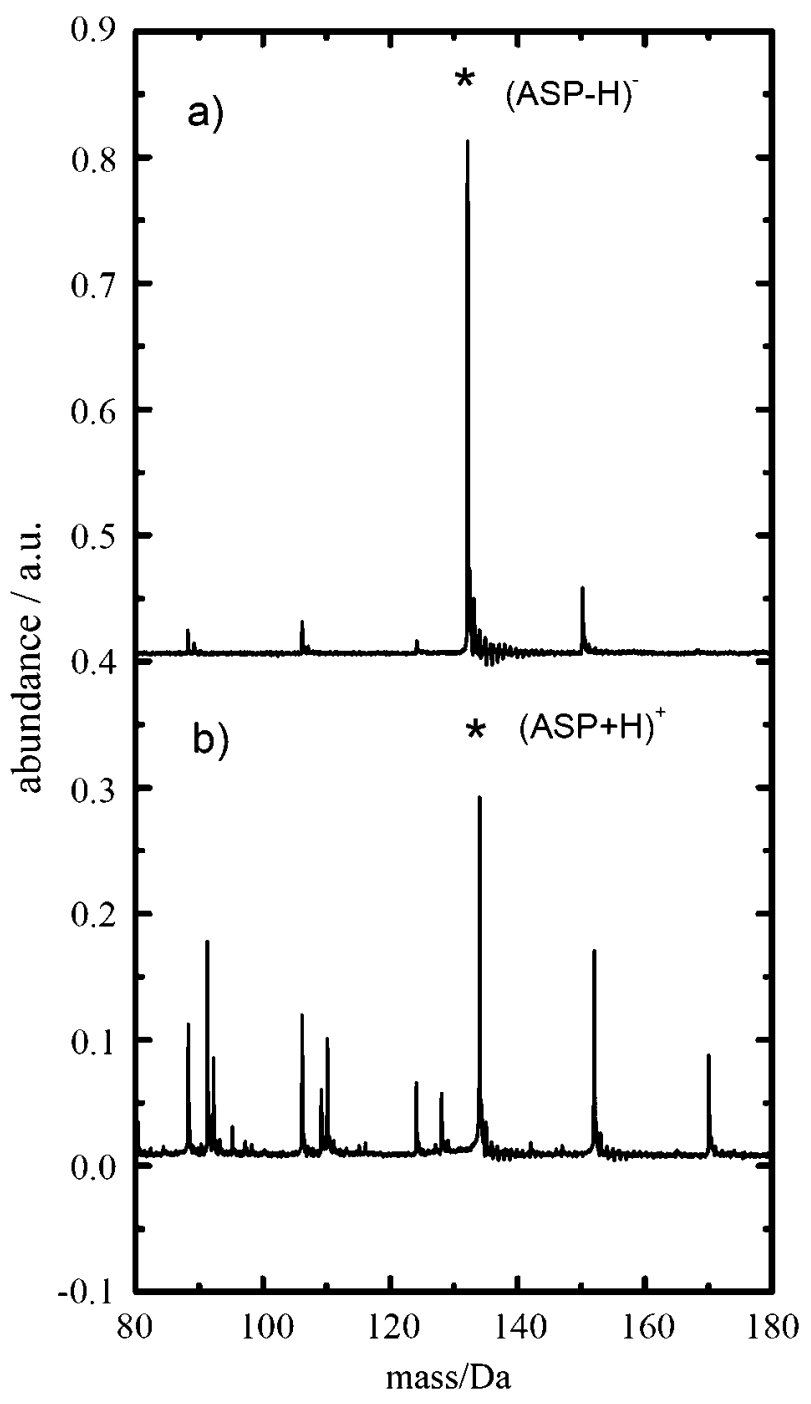

Fig. 6 Detection of aspartate in the negative (a) and positive ion mode (b). For more details see the text.

protonated or deprotonated water clusters ( $m<130 \mathrm{Da})$ and water aspartate aggregates $(m>140 \mathrm{Da})$. Another example close to the lysozyme example is shown in Fig. 7. Here spectra of cytochrome $\mathrm{c}$ in the positive (Fig. 7a) and negative ion mode (Fig. 7b) are recorded. Cytochrome $\mathrm{c}$ is a protein with many basic residues that can be detected in the positive and negative ion mode (as lysozyme it has a broad charge state distribution). In both cases only low charge states are detected with the +1 and -1 charge states (some contribution of the +2 charge state in the positive ion mode) being the most abundant. Although the charge state distribution is probably broad, the protein is again detected preferentially in the lowest charge states and the mass spectra do not reflect the actual charge state distribution in solution. However, the relative ion signals in both detection modes reflect the contribution of negatively and positively charged analyte molecules qualitatively. The ion signal in the negative ion mode is a factor of 5-10 smaller than the signal in the positive ion mode for cytochrome $\mathrm{c}$, which is easily understood due to the many basic amino acid residues. This situation changes, for example, 

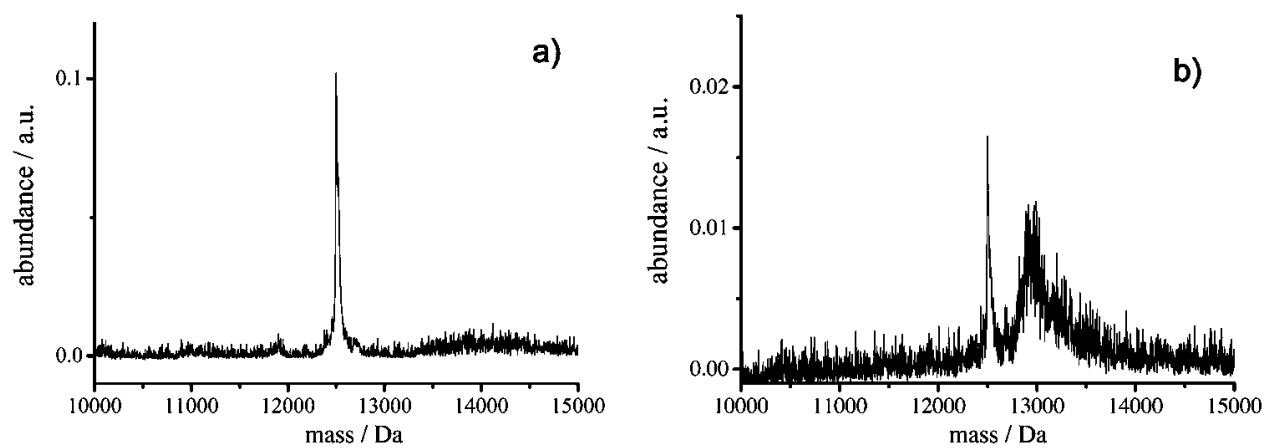

Fig. 7 Detection of cytochrome $\mathrm{c}$ in the positive and negative ion mode. (a) Positive ion mode. (b) Negative ion mode ( $\mathrm{pH}=7$, ammonium acetate buffer). The broad feature in (b) at $13000 \mathrm{Da}$ is likely due to protonated larger water clusters or aggregates.

for DNA strands which are usually negatively charged in solution, and which are best detected in the negative ion mode, as recently demonstrated by Brutschy's group. ${ }^{22,24}$ Nevertheless, it is also possible to detect DNA strands in the positive ion mode with a somewhat lower sensitivity. ${ }^{31}$

In summary, in our experiments we hardly monitor charge states larger than 2. This observation points to a general propensity of the IR-FL-MALDI-MS technique. (1) Usually low charge states of biomolecules are detected, depending somewhat upon the salt concentrations and the solvent. (2) In case of true ions only singly charged ions are easily desorbed from solution. Multiply charged cations (e.g., $\mathrm{M}^{2+}$ ) are usually desorbed and detected as singly charged aggregates and partially neutralized species containing $\mathrm{OH}^{-}$ (also in agreement with ref. 61). The simplest picture to explain these findings is that ion recombination is too fast for highly charged ions, leaving only singly charged complexes as "lucky survivors" in the desorption process. In addition, we want to note that we have no indication that the desorption process changes the oxidation number which is in contrast to the observations in ref. 61. We will discuss this issue in more detail in chapter 3 where experimental evidence of our conclusions is given. The low charge states in the experiment have an obvious advantage. Since there may be an ambiguity about singly charged monomer analyte molecules and doubly charged dimers (at the same $m / z$ value) we anticipate with IR-FLMALDI a dominating fraction of singly charged species. Since we can observe the abundance of dimers separately at the $\mathrm{m} / \mathrm{z}$ value of the singly charged dimer the uncertainty in general is only small. This feature is of importance for the detection of polymer distributions and the measurement of the aggregation kinetics of proteins with IR-FL-MALDI which is highlighted below.

\subsection{Towards a mechanistic understanding of IR-FL-MALDI}

The main effect of IR-FL-MALDI is that preformed ions or ionic aggregates can be isolated from the liquid (water) phase with a laser. Depending upon the laser conditions like the wavelength and, in turn, the penetration depth of the laser (see Fig. 2) as well as the energy deposit in the liquid, the whole volume or just the outer seam of the irradiated filament may be heated and disintegrated. Due to the short time-scale of the excitation the liquid filament may even be heated (at least in part) significantly above the critical point and the spinodal limit of metastable liquids. ${ }^{35,72}$ Although more than a decade passed after the effect was described first, ${ }^{19}$ the isolation of ions and ionic aggregates from liquid water (as for an ice or glycerol matrix in classical MALDI) is far from being understood. It appears clear that a direct ion evaporation from the liquid phase in thermodynamic equilibrium can be more or less ruled out due to the large solvation energy. On the other hand it is simple to show that the energy input in the process is much larger than that required for complete evaporation of the irradiated water volume, which should - in principle - be more than enough to eject molecules and molecular aggregates from the disintegrating matrix. ${ }^{31}$ Although a phase transition from liquid to gas phase appears reasonable, it is not clear, however, how the molecules are isolated and ejected at all from a hot water filament without destruction and how they are charged and, in turn, leave the process as a charged particle without ion recombination, which is a prerequisite for mass spectrometric analysis. Brutschy and co-workers have early tried to describe the LILBID process as a fast laser induced heating process of the irradiated liquid matrix volume above the critical point. In this simple and intuitive model the homogeneously and superheated phase is assumed to become supercritical and to expand rapidly after excitation resembling a supersonic hot seeded beam (Fig. 2). ${ }^{19}$ The ion yield in this process may be regarded as belonging to the fraction of "lucky survivors" of the fast ion recombination process in the gas phase. A similar model has also been adopted in ref. 21. Unfortunately, a general and quantitative theoretical framework and model for rationalizing and predicting ion yields in IR-FL-MALDI or LILBIDMS experiments has not evolved from these ideas yet. In order to estimate the ionization yield from an expanding supercritical phase expansion one has to make the approximation that the heating process is fast and sudden. The ion distribution in the dense supercritical gas is close to the Debye-Hückel ion distribution in the liquid before the excitation. In the early phase and during expansion, the density and the dielectricity constant of the solvent decrease. Because of the reduced shielding of the ions due to the solvent, cations and anions recombine to a large extent. The probability of recombination should depend upon the distance of the ions to their oppositely charged counter-ions. In solution at an early time the Debye screening radius is a first approximation for the average 
distance between the recombining ions. As displayed in Fig. $8 \mathrm{a}$, ions will recombine if their relative separation is small and those with a larger distance will be isolated in the process. Very likely, only a small fraction of the total ion concentration in solution $\left(10^{-4}-10^{-8}\right)$ can be isolated ${ }^{19}$ and, in turn, detected in the gas phase by mass spectrometry. The first quantitative calculations of ion recombination rates in a dense gas phase (for situations such as considered here) has been published by Baer and co-workers. ${ }^{55}$ If we make the approximation that the ion recombination (yield) in the gas phase depends upon the Debye screening length, ${ }^{31}$

$$
l_{D}=\sqrt{\frac{e^{2}}{\varepsilon_{0} \varepsilon k_{\mathrm{B}} T} \sum_{\alpha} Z_{\alpha}^{2} \frac{N_{\alpha}}{V}},
$$

( $Z_{\alpha}$ : charge state of ions, $N_{\alpha} / V$ : concentration of positive and negative ions, other symbols as in ref. 31 ) in the liquid phase

a)
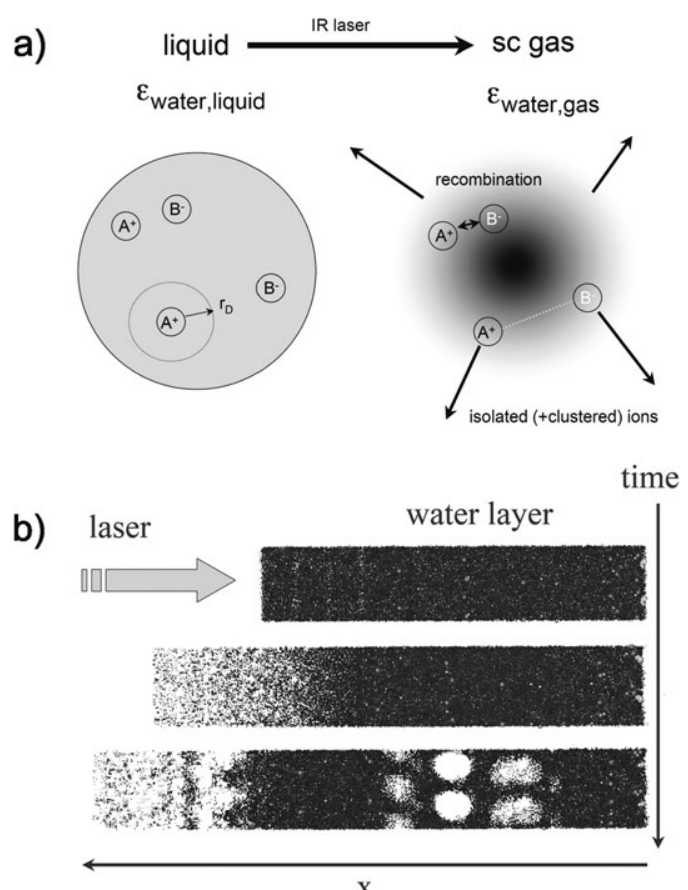

$\mathrm{X}$

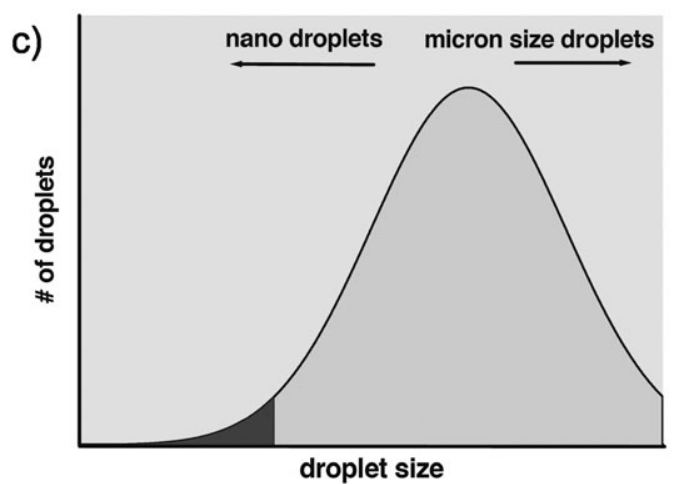

Fig. 8 (a) Supercritical phase expansion and incomplete ion recombination model. (b) Liquid dispersion of a liquid water phase with a laser (schematic). (c) Droplet size distribution in the laser induced liquid phase dispersion model (for more details see the text). before excitation, one may predict that the ion signal in the experiment depends upon the ion strength, the nature of the ions as well as their charge states, the solvent and its dielectricity constant and temperature. Although several predictions appear to agree qualitatively with experimental observations, e.g., the saturation of the ion signal for high concentrations, it is generally difficult to match all experimental observations quantitatively with the somewhat idealized model, which we may call supercritical phase expansion and incomplete ion recombination model (SPE-IIR). A somewhat related model has also been adopted later for the explanation of the ion yields in UV-MALDI experiments by Karas et al. ${ }^{66}$

The drawback of the above model is that quantitative predictions do not necessarily agree with all experimental observations. First of all, it is difficult to explain the striking linearity of the gas phase ion signal as a function of solution concentration over many orders of magnitude. It would rather predict decreasing ion signals as a function of solution concentration. In recent molecular dynamics calculations ${ }^{73}$ we have explored the effect of incomplete ion recombination for a supercritical phase expansion ('lucky survivor' model) and a model that we will call 'liquid dispersion model' (see below). It was found that charge separation via the latter mechanism is significantly more efficient than for the SPE-IIR model because gas phase ions are nearly quantitatively recombined after a very short time, and leaving hardly any 'lucky survivor', as expected.

In addition, the ideal assumption of a superheated homogeneous volume expanding as a pure gas very likely does not hold. Any heating process of a liquid microfilament is always accompanied by dispersion of the liquid (Fig. 2a, b) rather than a complete phase transition. The degree of dispersion ranges from isolated ions to larger droplets containing analyte and ions that do not contribute to the ion yield. Any excitation of a liquid microfilament produces a highly dispersed liquid driven by the hot gas phase, even at the laser-irradiated side due to re-condensation on a picosecond time-scale. ${ }^{56}$ In addition, inhomogeneous heating, spinodal decomposition, ${ }^{72}$ and shock wave formation produces a highly efficient dispersion of the liquid even at the back side of the liquid beam and a small fraction of nano-droplets that are small and hot enough to boil off the water and make it to the isolated ionic aggregates. Such a process does even require much less energy than complete evaporation. This situation is schematically depicted in Fig. 8b and $\mathrm{c}$. We have recently proposed a model that involves the picture of a dispersed liquid forming nano-droplets with embedded biomolecules and ions. ${ }^{31,32}$ As displayed in Fig. $8 \mathrm{c}$, most of the droplets generated in the dispersion are too large, they cannot contribute to the ion yield because they cannot boil off the water. A simple estimation shows that heated water droplets cannot evaporate easily in vacuum-i.e. without an additional source of heat. The heat of evaporation of water is $<40 \mathrm{~kJ} \mathrm{~mol}^{-1}(0.4 \mathrm{eV} /$ molecule $)$. For evaporation of an isolated droplet in vacuum that is still liquid the evaporation energy can only come from the internal energy of the droplet. Thus, it is unlikely that a larger hot liquid droplet is formed that evaporates completely in vacuum. This situation is certainly different from that for spray techniques where continuous evaporation takes place due to heating from 
a bath gas at high pressures. For the "ionization process" the only important requirement is that the droplets contain an excess charge and that the droplets are not too large. The evaporation of charged droplets pass their charge to the embedded biomolecules. The probability of finding an excess charge in a dispersed volume (nano-droplet) follows a Poisson statistics with significant deviations from that at high concentrations. ${ }^{73}$ It has been found that such a model naturally predicts a linear behavior of the ion yield as a function of liquid concentration over many orders of magnitude and a saturation at the highest concentrations. ${ }^{73}$ In IR-FL-MALDI mass spectrometry the dispersion of droplets must - at least in part-be so efficient, that nano-droplets are formed which are on the order of the size of the biomolecules they contain. The small fraction of nano-droplet ranging in size from a critical size down to the cluster and isolated molecule is displayed in Fig. 7c. The critical droplet size is related to the evaporation of the droplet, its temperature and the probability of finding an excess charge in the dispersed volume. The latter quantity may correlate with the Debye screening length $l_{\mathrm{D}}$. The simple picture that emerges from the dispersion model is that the dispersed nano-droplets must be smaller than the Debye screening radius. In such a case the dispersion can separate an ion from its counter-ion resulting in an excess charge in two nano-droplets. In the opposite case an ion pair is formed which does not produce a charged droplet. The model also naturally explains the observation and occurrence of low charge states, which are a direct consequence of the charge statistics in the dispersed droplets (liquid volumes). It is noted that the supercritical phase expansion and incomplete ion recombination model is just the limiting case for infinite dispersion of the liquid. Because any disintegration and even the production of a homogeneous supercritical phase is a dispersion of the liquid we have chosen the general acronym IR-FL-MALDI in which DI stands for dispersion of ions and ionic aggregates. We believe that the dispersion of the liquid into droplet sizes spread over many orders of magnitude is the key to understanding many of the key features of the IR-FLMALDI-MS technique. The model also naturally explains why we can observe ions desorbed from the back-side of the irradiated beam. ${ }^{32}$ Finally, we note that we measured the velocity of the desorbed ions and ionic aggregates. We usually determine velocities well above $1000 \mathrm{~m} \mathrm{~s}^{-1}$ which led us to propose a non-thermal shock wave desorption in ref. 31 and 32. These results are somewhat different than those reported in ref. 19 where significantly lower desorption velocities have been reported. We tentatively attribute this to different experimental conditions. Finally, it should be noted that the present model explains a number of features of IR-FL-MALDI-MS reasonably well, but some of them-such as, e.g., ion yields and desorption of surface active species - are beyond such a simple model.

\subsection{Linear response of IR-FL-MALDI-MS and probing the liquid phase}

The observed linear response of liquid beam desorption mass spectrometry, i.e., the striking linearity ${ }^{31-33}$ of the gas phase signal as a function of liquid concentration has been found in

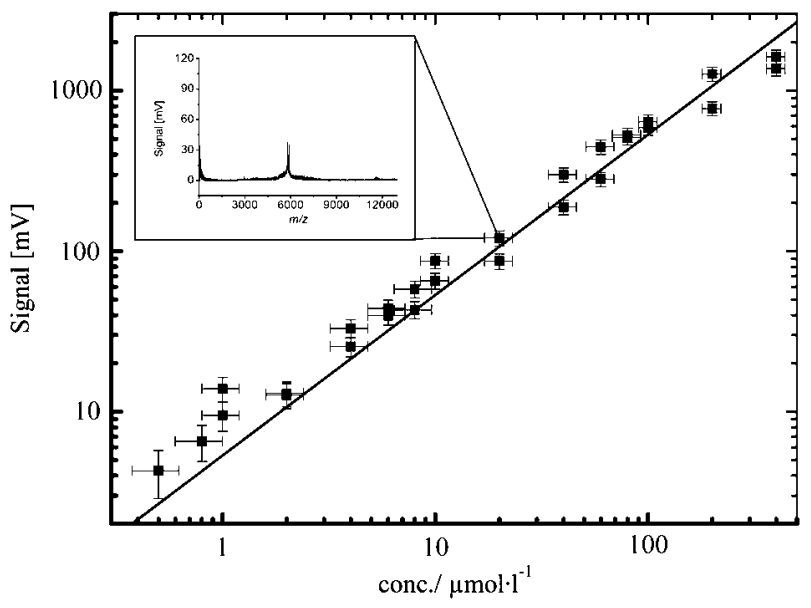

Fig. 9 Near-linear response of the insulin mass peak intensities as a function of concentration (pos. ion mode, $\mathrm{pH}=2-3$ ). The solid line shows an exact linear behavior. In the inset a mass spectrum is given for a single data point.

many of our experiments including a recent report by Brutschy's group $^{22}$ but not in ref. 61. A typical example (insulin) is displayed in Fig. 9. ${ }^{32}$ The linear response is mainly seen for lower concentrations up to concentrations on the order of $10^{-4}-10^{-3} \mathrm{M}$ (molar) for proteins or $10^{-1} \mathrm{M}$ for amino acids, depending upon the system under investigation. ${ }^{32}$ Beyond this linear region a more or less pronounced saturation is observed. $^{22,56}$ Noteworthy, the linear response in the doubly logarithmic plot is often not just a straight line but also a straight line passing the origin and having the slope of unity in favorable cases. The slope that may vary from 1 to 0.85 appears to depend upon the desorption conditions and, in turn, upon the degree of dispersion and, in turn, tupon he nano-droplet sizes. A somewhat similar behavior has also been seen in electrospray experiments, however, the linear response is often not as pronounced as that found for IR-FL-MALDIMS and the saturation sets in earlier. The relationship between signal intensity and original analyte solution concentration has been studied extensively there. ${ }^{74}$ It is known from the electrospray technique that in larger droplets, analyte surface activity, concentration, and competition from other species can affect overall ionization efficiency, the extent of ionization "suppression" and quantitation. A typical relation between ESI-MS response and solution concentration is characterized by two distinct regions. The first region, often referred to as the linear dynamic range, represents a concentration range from the lower limit of detection up to about $10^{-5} \mathrm{M}$, where the signal response increases linearly with increasing concentration. In a second region encompassing progressively higher concentrations, the signal intensity levels off and may finally even decrease as the concentration is further raised. A further complication is that the linear range, and the concentration where the transition into the "level off" region occurs, are different for ions of different charge state. Therefore, if one were to "calibrate" the ESI ion signal, one might have to do this for every charge state separately.

The origin of the linearity in IR-FL-MALDI-MS has been investigated theoretically recently. ${ }^{73}$ Assuming a dispersed 
liquid model in which nano-droplets are generated that make it to the isolated ion, the linear behavior may be rationalized. A good parameter - although only providing a qualitative guide - is the Debye screening range $1_{D}{ }^{75}$ that describes in a classical sense the average distance to the counter-ion in solution. The general rule of thumb for a linear response appears to be that the droplets generated in the dispersion have to be significantly smaller than the Debye screening length which is determined for a particular solution concentration. ${ }^{31}$

Let us assume that we inspect and measure (the contents of a sum of) $N$ droplets. For low concentrations most droplets do not contain any ions, although the Debye radius is larger than the droplet size. For droplets containing an ion the probability of charging is enhanced at low concentrations. For increasing concentrations, the probability for a charged droplet containing an excess ion increases linearly up to a point where the average number of ions in the droplets is close to unity. This is the ultimate limit of the linear response. For higher concentrations a saturation and even a decrease in the ion yield is observed. $^{73}$

\section{Applications of IR-FL-MALDI}

\subsection{Non-covalently bound complexes}

As shown in the previous chapters, liquid beam desorption mass spectrometry - as the ESI technique - can be regarded as a sensitive and soft technique for isolating intact covalently bound biomolecules from the liquid (water) phase. However, a real test for any method is the desorption or isolation of noncovalently bound complexes. Brutschy and co-workers have early shown the potential of LILBID for the analysis of noncovalently bound complexes. ${ }^{20,23,24,29}$ We will highlight here two examples, namely the Ribonuclease $\mathrm{S}$ and the hemoglobin complexes, which were investigated by Wattenberg et al. some time ago. ${ }^{29,30}$

A well understood and well studied system, but at the same time a nice benchmark for any soft desorption technique is hemoglobin. The protein complex is an assembly of four units - two $\alpha$-(15 125.3 Da) and two $\beta$-chains (15 865.2 Da) with four heme-groups - which are non-covalently bound to each other. $\alpha$ - and a $\beta$-chains first form a heterodimer and two heterodimers finally form the tetrameric hemoglobin complex. ${ }^{76}$ Important interactions between the sub-units are hydrophobic interactions and salt bridges. The hemoglobin is very sensitive to $\mathrm{pH}$ changes and heat, making it an attractive candidate for testing new mass spectrometric techniques. In the past, it has been investigated with ESI-MS (e.g., ref. 77) but not so successful with MALDI-MS. ${ }^{78,79}$ Fig. 10 displays mass spectra from our lab for different IR-FL-MALDI-MS desorption conditions $\left(5 \times 10^{-4} \mathrm{M}\right.$, ammonium acetate buffer). If the desorption energy is too high $(0.5 \mathrm{~mJ})$ only the monomers are seen in the mass spectrum (Fig. 10b). Although there is significant background possibly from the water matrix, the resolution of the spectrometer is high enough to distinguish the apo- $\alpha$, holo- $\alpha$, apo- $\beta$, and holo- $\beta$ units. If the same laser pulse energy is used (Fig. 10a), but with the micro-beam moved slightly out of the focus of the IR laser beam, the
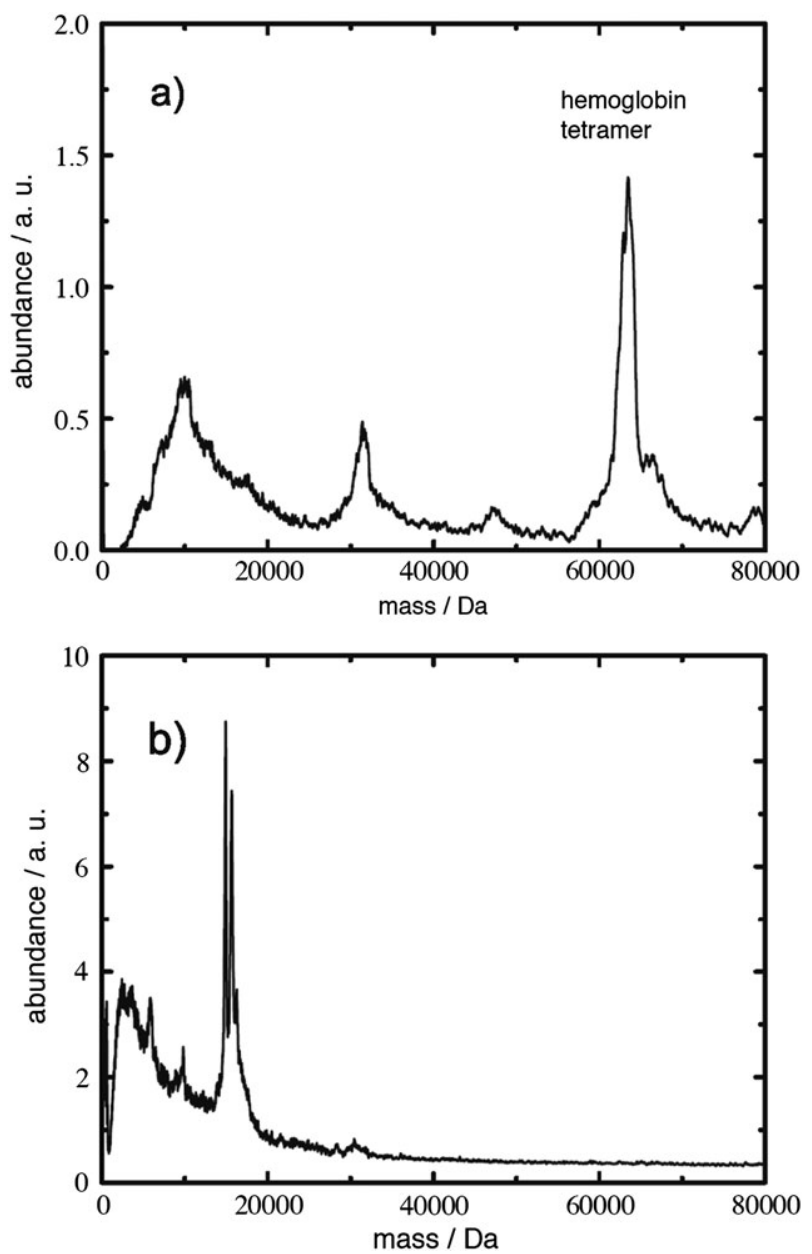

Fig. 10 Desorption of the hemoglobin complex in water (pos. ion mode, $\mathrm{pH}=7$, ammonium acetate). (a) Desorption of the complex with $0.5 \mathrm{~mJ}$ focused light, but the water filament moved slightly out of the laser focus. Note, nearly exclusively the dimer and the tetramer are detected. Compare also results from ref. 30. (b) Same experiment but laser focused tightly on the water filament. In this case no complex is detected.

hemoglobin tetramer as well as the dimer can be observed in the positive ion mode (see also ref. 30). Unfortunately, as in the following example below, the resolution is poor probably due to water aggregation, which however may stabilize the complexes efficiently. More IR laser energy would probably enhance boiling-off of water from the complexes but obviously also complex dissociation. For weakly bound complexes obviously a compromise between efficient desorption and sufficient desolvation has to be found.

Ribonuclease S (RNaseS) is a non-covalently bound protein-peptide complex with a known structure and mass (11 534.0 and 2166.3 Da). The RNaseS complex has been investigated extensively in the past and it has been found that the stability of the complex depends strongly on temperature and solution $\mathrm{pH}^{80-85}$ The highest stability is found at the melting point of ice $(273 \mathrm{~K})$. For the binding of the protein and the peptide hydrophobic interactions and hydrogen bonding play a decisive role, and the strong temperature dependence of the complex stability, as reflected in the temperature 
dependence of the equilibrium constant, is attributed to the flexibility of the protein backbone and the peptide chain. The strong dependence of the stability of the complex on temperature and $\mathrm{pH}$ has made this system an interesting candidate for MALDI- and ESI-MS studies. In Fig. 11 a series of mass spectra in positive ion mode for different desorption conditions for RNaseS in water at a concentration of $10^{-5} \mathrm{M}$ are displayed. The high-pressure inlet system and the nozzle have been temperature stabilized at $274 \mathrm{~K}$. While Fig. 11a displays the result for a moderate desorption energy $(0.4 \mathrm{~mJ})$ at $\mathrm{pH} \sim 7$ (ammonium acetate buffer), Fig. 11b gives the corresponding data for $\mathrm{pH}=2$ and the same laser energy. No complex is seen
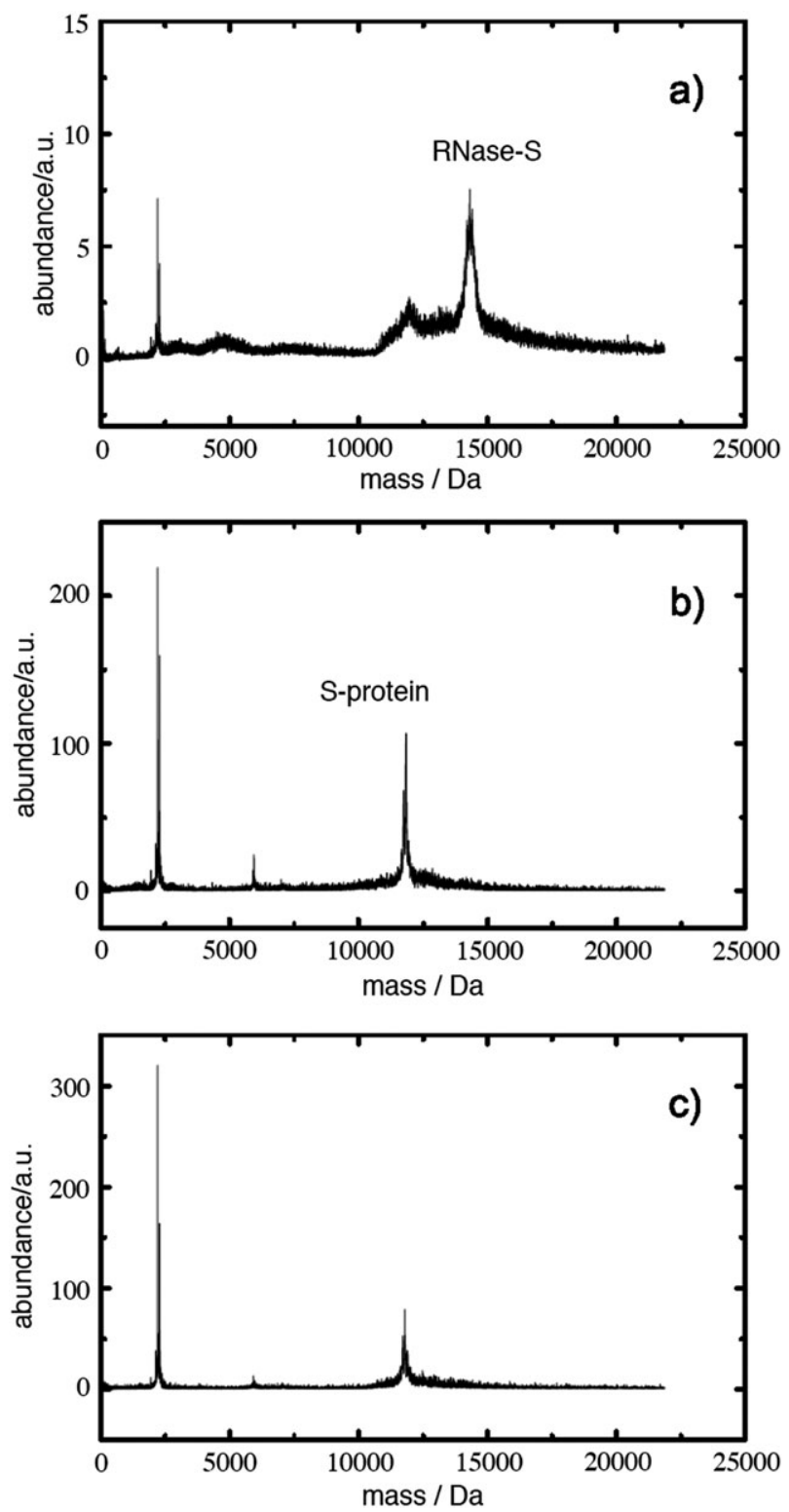

Fig. 11 Desorption of the ribonuclease S complex (RNase-S) illustrating the softness of desorption and the dependence of the results upon laser and solution conditions such as $\mathrm{pH}$ (pos. ion mode, ammonium acetate buffer). (a) Desorption with low desorption energy $(0.4 \mathrm{~mJ})$ at $\mathrm{pH} 7$, (b) desorption for $0.4 \mathrm{~mJ}$ at $\mathrm{pH}=2-3$, (c) desorption for $0.6 \mathrm{~mJ}$ laser energy at $\mathrm{pH}$ 7. Only in the first experiment (a) the complex can be detected. due to the low $\mathrm{pH}$. In Fig. $11 \mathrm{c}$ results for $\mathrm{pH}=7$ and a relatively high desorption laser energy $(0.7 \mathrm{~mJ})$ are displayed. In this case the complex is also not observed due to the sensitivity of the complex towards temperature. For higher initial solution temperatures the complex peak is also not detectable, a result that may show that the technique monitors to some extent the situation in solution. Although the complex can be observed with liquid beam desorption MS some complex dissociation is unavoidable making this technique not necessarily superior in our lab to ESI and MALDI with regard to the "softness" of desorption in this particular case.

Another nice example of the power of liquid beam desorption mass spectrometry has also been reported from Brutschy's group. They have desorbed short synthetic DNA double strands from water solution ${ }^{24}$ providing firm evidence that IRFL-MATRIX-MS (LILBID-MS) is not only a soft desorption technique but also provides at least semi-quantitative information of biomolecular aggregates in solution. A much-studied DNA single strand is the Dickerson dodecamer DD. Since it is self-complementary it serves as a model helix to test the specific binding of ligands in the minor groove of the duplex DNA. $^{22}$ In a very recent paper the same group has reported experiments in which the LILBID technique has been used to investigate specific interactions with minor groove binders such as netropsin and Distamycin A interacting preferentially with AT-rich regions of the DNA. These ligands are of interest due to their anticipated anti-tumor activity. In both cases the DNA complexes have been detected in the negative ion mode.

\subsection{Biokinetic applications}

The enormous potential of mass spectrometry for the investigation of the kinetics of solution-phase reactions was recognized early. ${ }^{86}$ Therefore, mass spectrometers equipped with electrospray ionization sources have been employed to follow kinetics of a (bio)chemical process "triggered" by mixing techniques and by direct injection of the reaction mixture into the ion source. ${ }^{87}$ Also, the use of ionization techniques other than ESI for kinetic MS studies has been explored by a number of groups (e.g., ref. 88 and 89) A good overview of mass spectrometry in biophysics in general and for the measurement of biokinetics in particular is given in the book by Kaltashov and Eyles. ${ }^{90}$ In this section we highlight typical applications of the IR-FL-MALDI-MS technique. Due to the linear response and the continuous mode of the technique the approach is ideally suited for kinetic and mechanistic studies and a determination of rate constants in biochemical systems. Although it is known that the IR-FL-MALDI-MS technique, in principle, depends on the analyte concentration, total ionic strength, counter-ions, surface active molecules, and so forth, such that the ion signal in kinetic measurements may be perturbed or, at least, influenced (even in a non-linear fashion), we have not found large interferences for most systems although we searched for it. We do usually vary the relevant concentrations in order to trace and exclude such interferences. As highlighted in section 2 the IR-FL-MALDI-MS technique is actually rather insensitive to these effects if other possibly perturbing species are not present in large excess. However, even for minority species in a large excess of other 
molecules we have found a near-linear response for the minority species, a feature that is discussed in section 3.4.

3.2.1 Protein aggregation and amyloid fibril formation. Neurodegenerative diseases such as Alzheimer's, Parkinson's and the transmissible spongiform encephalopathies (TSEs) are characterized by abnormal protein deposits, often in the form of highly symmetric amyloid fibrils. ${ }^{91}$ Amyloid fibrils are polypeptide aggregates in which the polypeptide backbone is arranged in a specific and characteristic cross- $\beta$-sheet quaternary structure. In the past couple of years there have been reports about amyloid formation of non-pathogenic proteins not assigned to any disease. ${ }^{91}$ This led to the conclusion that amyloid formation is no specific property of only a few proteins and polypeptides, namely those that have been described to assemble into clinical amyloid deposits. Instead, is has been found that many different systems are readily able to form amyloid fibrils in competition with normal folding, a process that is accelerated by autocatalysis (Fig. 12). However, the mechanism(s) of fibril formation in general are still not known very well and are currently the subject of intense research.

The physiological and therapeutic importance of insulin, and the fact that insulin easily aggregates in vitro, have made the hormone an excellent model for protein aggregation studies. ${ }^{92}$ The insulin aggregation is promoted under conditions favoring partly destabilized monomers and dimers, such as low $\mathrm{pH}$, high temperature and, to some extent, contact with hydrophobic media. Insulin fibrils display characteristic features common to all amyloids: X-ray diffraction patterns; affinity to Congo Red and Thioflavin T; birefringence; biological dysfunction and high stability against temperature; pressure; low $\mathrm{pH}$; and proteases. ${ }^{93}$ Considerable debate exists about the mechanism of fibril assembly in general and for

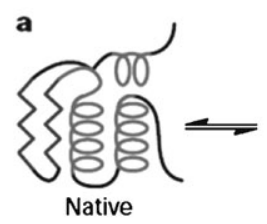

$$
\text { b }
$$
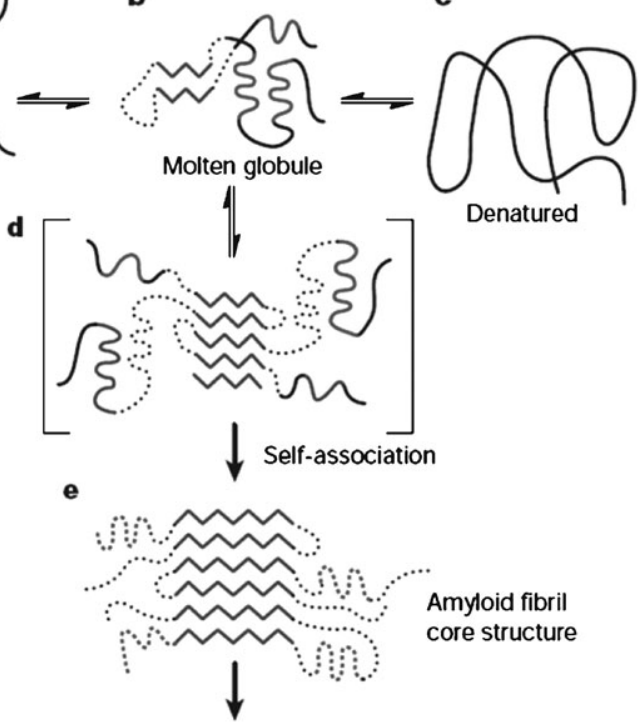

Further assembly of protofilaments

Fig. 12 Folding path and misfolding pathway of a protein leading to the native state or a fibril. insulin in particular and about the existence and nature possible of intermediate species. ${ }^{32,94}$

In order to shed more light on the mechanistic features of protein aggregation mechanisms for insulin we have employed time-resolved IR-FL-MALDI-MS. ${ }^{32,95}$ An advantage over alternative mass spectrometric approaches is its near-linear response, which facilitates kinetic measurements and the determination of rate constants. It is well known that insulin aggregation can be initiated by heat and low $\mathrm{pH}$. Therefore, aqueous solutions of insulin at $\mathrm{pH}=2$ in the concentration range between $10^{-5} \mathrm{M}$ and $5 \times 10^{-4} \mathrm{M}$ were heated at $70{ }^{\circ} \mathrm{C}$ (weakly stirred). Samples of the solution were injected in the liquid beam source and analyzed in the TOF mass spectrometer as a function of time. Typical experiments are displayed in Fig. 13. As is obvious from Fig. 13a the parent insulin mass peak at $t=0$ decreases within the course of the experiment until it completely vanishes for longer times. If the intensity of the insulin mass peak is plotted against the time, typical sigmoidal traces are obtained (Fig. 13b). The initial lag time corresponds to a plateau in the signal trace, which decays rapidly to zero after a so-called lag time. Such traces are characteristic of the kinetics of amyloid systems. The lag time in many systems is pronounced. If the lag time becomes smaller, the sigmoidal trace resembles more and more an
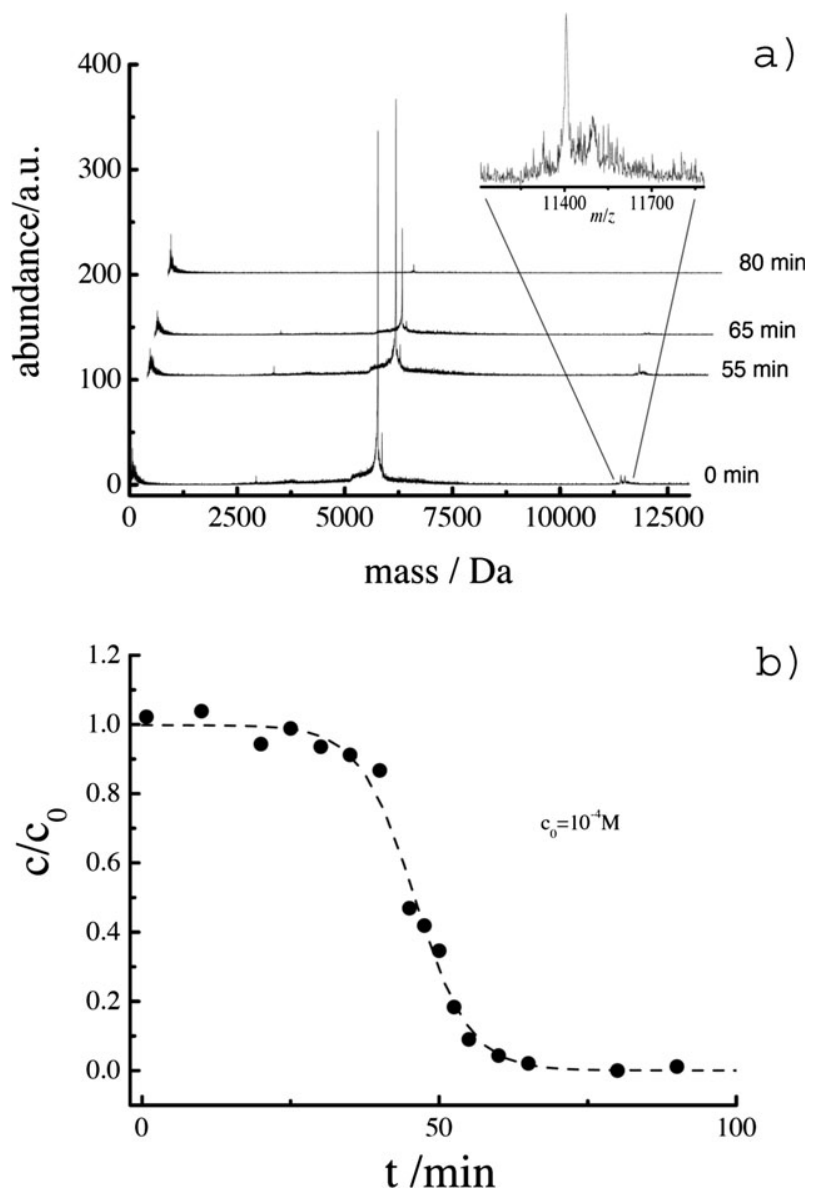

Fig. 13 (a) Time dependent spectra of insulin. (b) Aggregation kinetics of insulin, as measured on the mass peak of the parent insulin (pos. ion mode, $10^{-4} \mathrm{M}, \mathrm{pH}=2-3$ ). 
exponential decay. For short and intermediate times also mass peaks of insulin multimers are detected (Fig. 13a) which are likely off-pathway multimers and not intermediates in the fibril assembly. ${ }^{32}$ Their concentration is in general quite low and vanishing for longer aggregation times. In the present experiments the kinetics has been followed via the monomer concentration, i.e., the concentration of soluble protein. It is known that insulin forms fibrils and amorphous aggregates depending upon the temperature, the ions in solution (acid), concentrations, etc. For the present conditions not very long fibrils are expected. The morphology of the aggregates was monitored with the in situ atomic force microscopy. Typical fibrils and aggregate structures in water solution are shown in Fig. 14a where a $385 \times 385 \mathrm{~nm}$ area with short fibrils is displayed. $^{32,95}$ The features of the kinetic traces such as Fig. 13 strongly suggest that the formation of amyloid fibrils may be - in the simplest case - a two-step reaction that consists of a slow reversible nucleation step (until a critical aggregate size is reached) followed by a faster molecular assembly step. Somewhere along this mechanism a transition between native or
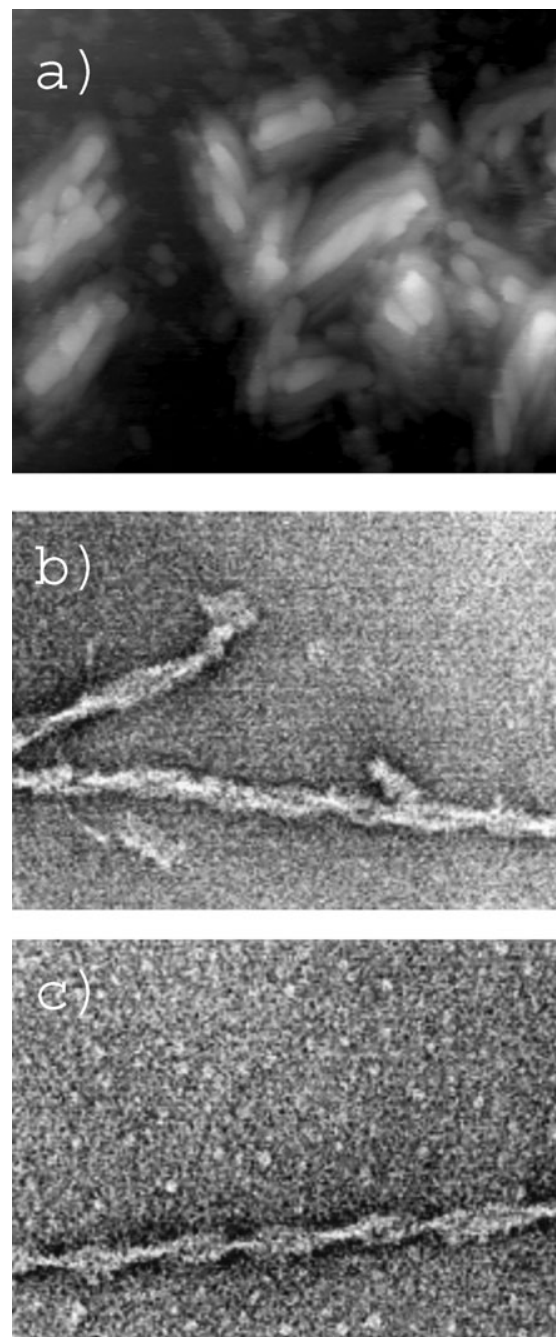

Fig. 14 (a) AFM micrographs of insulin fibrils (adapted from ref. 32). (b) EM micrographs of the morphology of tau protein fibrils with added hepatin. (c) tau protein fibrils without adding hepatin (adapted from ref. 101). partially unfolded conformations to the well-known specific $\beta$-sheet conformation within the fibril takes place. Interestingly, seeding of preformed fibrils diminishes the lag phase of the nucleation step, ${ }^{32,95}$ suggesting that the nucleation step plays an important role (rate determining step) in the kinetics of fibril formation.

Currently, there are three popular mechanistic models describing amyloid fibril formation (Fig. 15). ${ }^{96}$ The first is referred to as template assembly (TA), wherein a pre-assembled nucleus binds to a soluble state $(\mathrm{S})$ peptide in a random coil conformation in a rapid pre-equilibrium step. This is followed by a rate-determining structure change to add the peptide to the growing end of the fibril or filament, presumably as part of the $\beta$-sheet-rich quaternary structure. The second model is the monomer directed conversion (MDC) model, which implies that a monomeric peptide can adopt a conformation called the A state that is analogous to the conformation adopted in the fibril. In the rate determining step this structured monomer (A) then binds and converts an S state monomer, resulting in an A state dimer. The dimer then dissociates and the structured monomers rapidly add to the end of the growing fibril (Fig. 15). The third model, the often invoked nucleated polymerization (NP) mechanism, is characterized by the rate limiting formation of a nucleus resulting from an equilibrium between monomers that are and are not assembly competent. Once a nucleus is established, assembly occurs by the addition of assembly competent monomers to the growing end of the fibril (i.e., the nucleus).

We found that for insulin none of the established models explains all experimental observables satisfactorily. Based upon our experimental findings for the insulin system we assume that monomers are loosely aggregated together to form micellar structures. ${ }^{95}$ A nucleated conformational conversion forms protofibrils, which assemble into larger filaments or which add more monomers and which serve as templates (see mechanisms in Fig. 15). Such a mechanism has been described by Serio et al. who also had problems to explain their aggregation results of Sup35 prion protein with existing models and termed the new model "nucleated conformational conversion (NCC)". The new aspect is that it invokes oligomeric intermediates for initiation and assembly. ${ }^{97}$ This mechanism is the reason why the aggregation of proteins in both cases described here can only be followed by monomer loss. Intermediate multimers are either not present, too large in mass or in too low concentration. The fact that we do not observe multimers like in a normal polymerization reaction is a characteristics of the aggregation mechanism which is distinctly different to ordinary polymerization. Therefore, we do not expect here to observe molar mass distributions such as observable for polymers (see section 3.3). This also holds for an other protein - the tau protein-investigated recently in our group.

The tau protein ${ }^{98}$ is a mainly but not exclusively neuronal microtubule-associated protein (MAP). One of its functions is the stabilization of axonal microtubules; ${ }^{99}$ other functions include a role in signal transduction, interaction with the actin cytoskeleton, neutite outgrowth, interactions with the plasma membrane, anchoring of enzymes such as protein kinases and phosphatases, and the regulation of intracellular vesicle 
Monomer-Directed Conversion (MDC)

A State Monomer

$S$ State Monomer
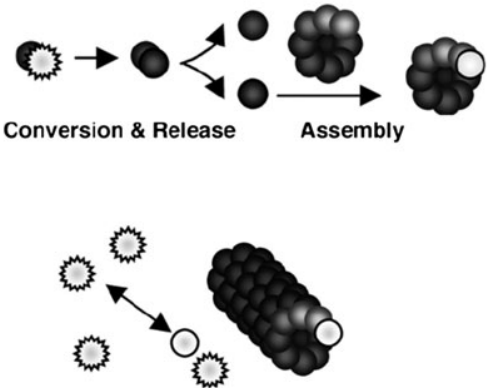

A State Stabilized by Interaction with Nucleus

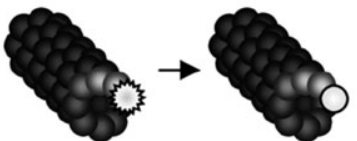

Conversion Coincident with Assembly

Fig. 15 Different kinetic models that have been invoked to explain the kinetics of protein aggregation and fibril formation of amyloid systems (adapted from ref. 97).

transport. ${ }^{98}$ Since tau is a highly soluble protein, which survives heat, denaturing agents or acid treatment without losing its biological function (the binding to microtubules and the stimulation of their assembly), it is counterintuitive that this protein should aggregate into insoluble fibers. Over the past couple of years a number of studies on the structure and assembly of tau, its phosphorylation by various kinases, and its interaction with microtubules have been carried out. ${ }^{98,100,101}$ Phosphorylation tends to dissociate tau from its natural partner, the microtubule. Since this increases the soluble pool of tau it might be an important first step in generating protein for the assembly of paired helical filaments (PHFs). However, the assembly itself appears to depend mainly on other factors (conformation, oxidation, nucleation by other components).

A general mechanistic picture of tau aggregation and fibrillation does not yet exist, although some progress has been made. ${ }^{100}$ It is assumed that the tau monomers dimerize either covalently by oxidation (disulfide bridge formation, stable dimer) or non-covalently (weakly bound dimers). The dimer appears to be the effective building block of PHFs. Nucleation is slow in the absence of polyanions but strongly accelerated in their presence (e.g., heparin) Elongation (with or without exogenous seeds) is slow in the absence of polyanions or by prevention of dimerization, indicating that a dimer-polyanion complex is incorporated into PHFs. Excess of polyanion is inhibitory. The stochiometry and the arrangements of monomers are also not known. ${ }^{98}$ The development of a quantitative assay for PHF formation using thioflavine $\mathrm{S}$, which is known to stain not only amyloid but also PHFs, opened a first possibility to a more detailed study of the mechanism of PHF formation. ${ }^{100}$

Parallel to the investigations of the aggregation of insulin we have applied IR-FL-MALDI-MS to monitor the aggregation and assembly of tau into PHFs. Recombinant tau protein was recovered and characterized as described in ref. 98 and 101. In a recent study four tau constructs including the wild type
HTau $40^{101}$ and the fragment $\mathrm{K} 18 \Delta \mathrm{K} 280^{101}$ have been investigated with the liquid microbeam MS-technique. ${ }^{102}$ As in the case of insulin we monitored the monomer (and dimer) protein peak as a function of time. At longer times fibrils are formed and have been identified via electron microscopy (Fig. 14). It has been found that the morphology depends somewhat upon the solution conditions and the chemicals added. Fig. 14b and c display two EM micrographs of fibrils obtained with and without heparin. The name PHFs is derived from their electron microscopic appearance as two strands. ${ }^{101}$ Fig. 16 displays a typical set of experiments in which the aggregation of the K18 $\Delta \mathrm{K} 280$ tau variant ${ }^{101}$ has been measured. Again, as in the case of insulin, a plot of the tau fragment mass peak as a function of time yields a sigmoidal trace. The kinetics of aggregation of HTau 40 and the $\mathrm{K} 18 \Delta \mathrm{K} 280$ variant has been measured as a function of time at $40{ }^{\circ} \mathrm{C}$ in the presence of DTT and DTT/heparin (see Fig. 17a, b). While the kinetics of the $\mathrm{K} 18 \Delta \mathrm{K} 280$ variant displays the typical sigmoidal time

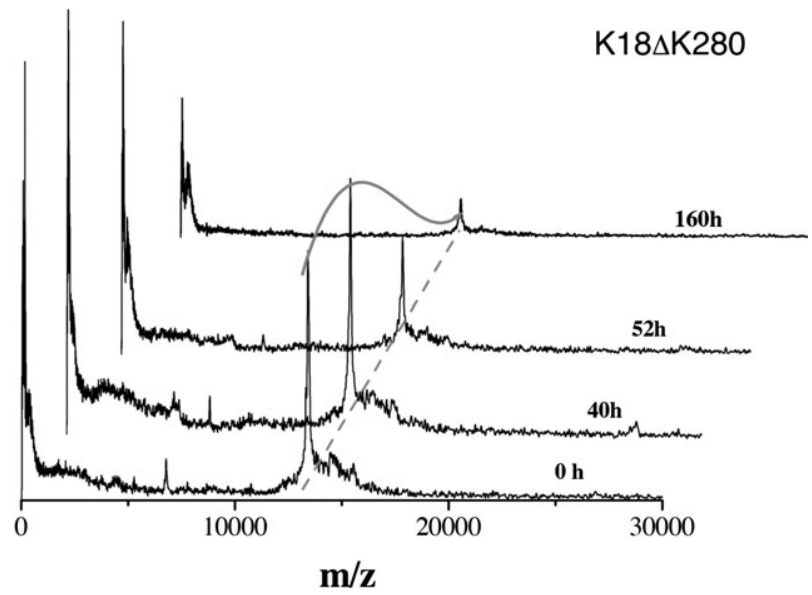

Fig. 16 Time dependent mass spectra during the aggregation of the tau-protein in water (positive ion mode). For more details see the text. 
a)

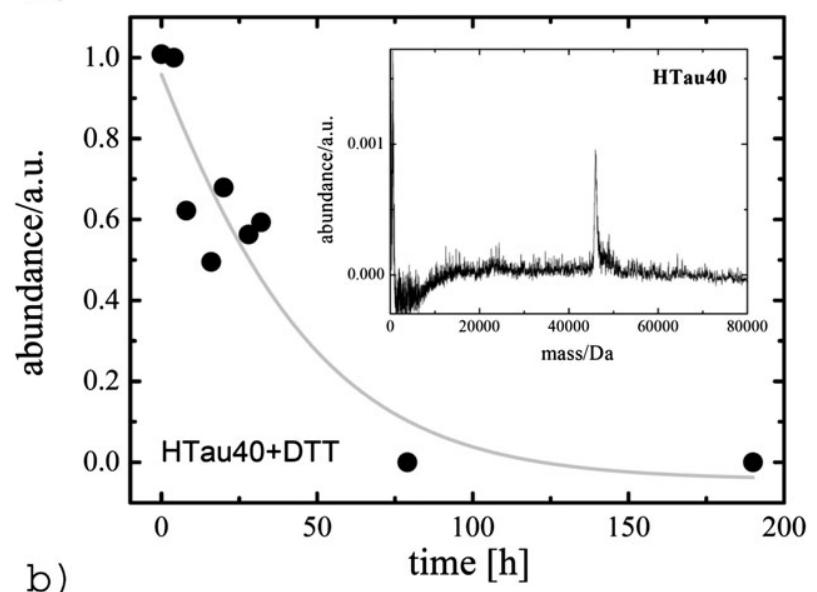

b)

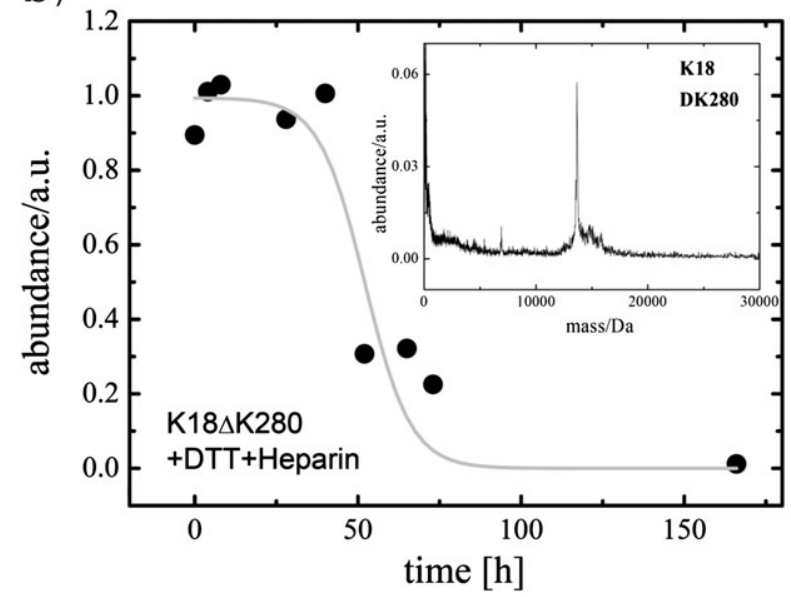

Fig. 17 Kinetics of the aggregation of different variants of the tauprotein: (a) wild type Htau40; (b) fragment K18 18280 . Inset: mass spectra of tau at $t=0$ (positive ion mode). For more details see the text.

evolution the HTau40 variant displays a nearly-exponential decay only, without any lag time. For the HTau40 variant the aggregation is even fast without addition of heparin and DDT does not slow down this process. The features of the kinetic traces such as in Fig. 17 (in particular Fig. 17b) strongly suggest that the tau aggregation is a two-step reaction that consists of a slow reversible nucleation step (until a critical aggregate size is reached) followed by a faster molecular assembly step (such as that found for insulin) according to Fig. 15. It is interesting to note that for the $\mathrm{K} 18 \Delta \mathrm{K} 280$ variant the kinetic time-scale measured via IR-FL-MALDI-MS is much slower than that measured with the thioflavine $\mathrm{S}$ assay. ${ }^{101}$ Since the kinetics measured here monitors the decay of the soluble monomer, the question now is what the thioflavine $\mathrm{S}$ assay measures under the same conditions. If the differences are not due to different solution conditions and/or protocols (which we tried to avoid carefully) it would be interesting to resolve this discrepancy. In any case, IR-FLMALDI-MS may be a powerful assay for these types of investigations. ${ }^{103}$ The aggregation of tau in this particular case could only be observed at the monomer and the dimer mass. ${ }^{102}$ Larger multimers are well above $100 \mathrm{kDa}$ and therefore invisible in our experiment. We would like to emphasize here that also in the present case we do not expect molar mass distributions such as those observed for synthetic polymers. Due to the special assembly mechanism of fibrils those cannot be detected or they have very low concentrations. Usually IRFL-MALDI-MS is actually a powerful technique for monitoring weakly bound multimers if they are present in solution. From the fact that we cannot detect them we conclude that the NCC mechanism also applies for the aggregation of tau.

3.2.2 $\mathrm{H} / \mathrm{D}$ exchange in weakly perturbed proteins-near equilibrium states of proteins. Protein dynamics and near equilibrium states of proteins may be investigated via isotopic H-D exchange. ${ }^{104}$ On the other hand, the conformational state in a qualitative sense may be detected from isotope exchange experiments. ${ }^{86,90}$ It has been demonstrated that time resolved mass spectrometry in combination with fast mixers and pulselabeling methods may follow and shed light on the folding and unfolding processes of proteins. ${ }^{87}$ Beyond protein folding investigations, an attractive feature of time-resolved mass spectrometry is to find solvent conditions where partially folded states become populated, at least transiently, at equilibrium. Such information is crucial for the investigation of mechanisms of protein aggregation, such as that highlighted above. As outlined below, it is highly likely that at least partial unfolding is important for amyloid fibril formation. However, it is still not clear how such partially unfolded and/or partially refolded molecules associate into amyloid fibrils. ${ }^{91}$ A number of proteins when mildly destabilized assume a kind of liquidlike state that has been discussed in terms of a form, named molten globule, thought of to be intermediate in structure between the native and unfolded states (see Fig. 12). It has frequently been suggested that the building block of amyloid fibrils under aggregating conditions is a molten globule protein. ${ }^{86}$

Under mildly denaturing conditions, such as altered $\mathrm{pH}$, temperature, or chaotrope concentration, protein stability can be altered such that, although the native state remains predominant, partially unfolded conformations also become populated. These more open structures are more prone to exchange with bulk solvent either due to weakening of hydrogen bonding interactions or due to exposure of residues that would normally be buried in the native protein. Using MS, we can indirectly observe these intermediates populated transiently under equilibrium conditions by the nature of their different amide hydrogen exchange properties of the protein.

lysozyme possesses a total of 255 labile (exchangeable) hydrogens, 126 from the backbone amide linkages, 126 from side chains and 3 from termini. Most of the side chains are located on the protein surface, and these together with the more accessible ones at the backbone exchange orders of magnitude faster than those buried in the interior of the protein. ${ }^{105}$ While the measurement of the H/D exchange at faster time-scales measures mainly the accessible surface hydrogens, H/D exchange at longer time-scale enables us to study the slower dynamics and thus more the overall structure of the protein. The slow exchange is indeed mediated by structural fluctuations of the protein that provide transient access to the solvent or the exchange agent. The slow exchange of buried sites can follow two mechanisms; the penetration 
mechanism where transient fluctuations of the protein chain in solution allow the solvent to diffuse inside the buried core, and the local unfolding mechanism. ${ }^{106,107}$ While the former is considered as a low activation process, the latter requires a higher activation energy. ${ }^{104}$ Hydrogen exchange rates for unprotected hydrogens have been measured extensively in the past as a function of $\mathrm{pH}$ and temperature. ${ }^{108}$ Two limiting cases arise depending on the following conditions: if the protein's structural dynamics (e.g., refolding or opening a local structure) is slow as opposed to the intrinsic exchange rate, $k_{\mathrm{ex}}$, all hydrogens in the transiently accessible segment will undergo exchange before refolding or closing of the local structure occurs. This case is known as the $\mathrm{EX}_{1}$ exchange mechanism or correlated exchange. ${ }^{109}$ If, on the other hand, the dynamics or refolding is much faster than the intrinsic exchange rate, we enter the regime of the $\mathrm{EX}_{2}$ mechanism, ${ }^{109}$ in which the dynamics or refolding occurs before all hydrogens involved or accessible in the dynamics are exchanged. Whether the exchange occurs via a correlated or uncorrelated hydrogen exchange mechanism (i.e., $\mathrm{EX}_{1}$ or $\mathrm{EX}_{2}$ ) may be determined from the mass spectra. While the $\mathrm{EX}_{2}$ kinetics often shows a simple peak shift smoothly in time, a more or less unambiguous evidence for a $\mathrm{EX}_{1}$ kinetics is the appearance of multimodal distributions of different species visible as in satellite peaks or broadened peaks. ${ }^{110}$

In order to explore near-equilibrium states of lysozyme in solution at $\mathrm{pH}=7$ in the temperature range between 0 and $85{ }^{\circ} \mathrm{C}$, investigation was conducted with time-resolved IRFL-MALDI-MS. ${ }^{11}$ Chicken egg white lysozyme lyophilized powder (Sigma) was dissolved in $\mathrm{D}_{2} \mathrm{O}$ and incubated for $10 \mathrm{~h}$ at $50{ }^{\circ} \mathrm{C}$. Then it was allowed to cool down to room temperature before being frozen and lyophilized. For the experiment the protein was quickly but very carefully dissolved in $\mathrm{H}_{2} \mathrm{O}$ and injected in the liquid beam MS source. Typical mass peaks, shifted as a function of time, are displayed for $50{ }^{\circ} \mathrm{C}$ in Fig. 18. For small exchange times the peak is broadened and is shifting towards lower masses, due to the back exchange of hydrogens. With the shift, also a narrowing of the peak width appears to be visible, which may be attributed to a change in the mechanism from $\mathrm{EX}_{1}$ at small reaction times to the $\mathrm{EX}_{2}$ mechanism. In Fig. 19 the kinetic traces are shown for different temperatures on a logarithmic time axis plot. We want to emphasize that the first data point can be taken at about $25 \mathrm{~s}$ after mixing and injection such that the experiment in the present configuration cannot monitor relatively fast events (see the shaded area in Fig. 19). We anticipate that during this time already one-half of the exchangeable (surface) hydrogens are exchanged and thus invisible and therefore only the slowly exchanging ones are visible in the present variant of the experiment. From the time traces in Fig. 19 it is clearly visible that at a temperature of $0{ }^{\circ} \mathrm{C}$ the protein is in a stable native state ${ }^{105}$ that slowly exchanges hydrogens due to molecular motion and dynamics at this low temperature. As the temperature is increased the protein becomes increasingly instable and the exchange rate increases. At the melting temperature of lysozyme the exchange is too fast to be measured at all. With experiments like the latter, one may obtain information about conformations of the protein under destabilizing conditions, e.g. in lysozyme aggregation experi-

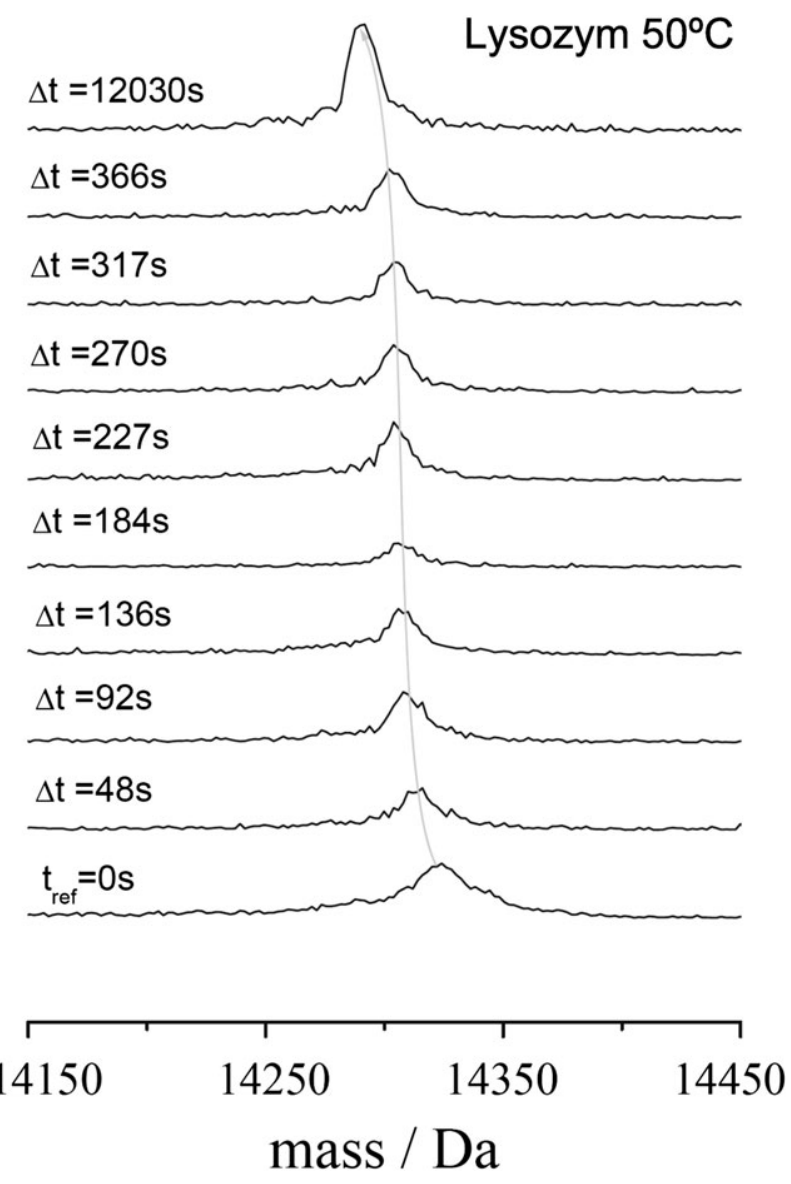

Fig. $18 \mathrm{H} / \mathrm{D}$ exchange of lysozyme as a function of time at $50{ }^{\circ} \mathrm{C}$ (positive ion mode). The increased peak width at early times is likely due to multiple species broadening the spectrum, as opposed to the linewidth at later times which appears to be broadened only by the intrinsic isotope distribution of lysozyme.

ments. Such near equilibrium states are visible as plateaus in plot like Fig. 19. ${ }^{104}$ At present we are working on a combination of fast mixers to the current setup, which will enable us to measure exchange processes even on the millisecond timescale.

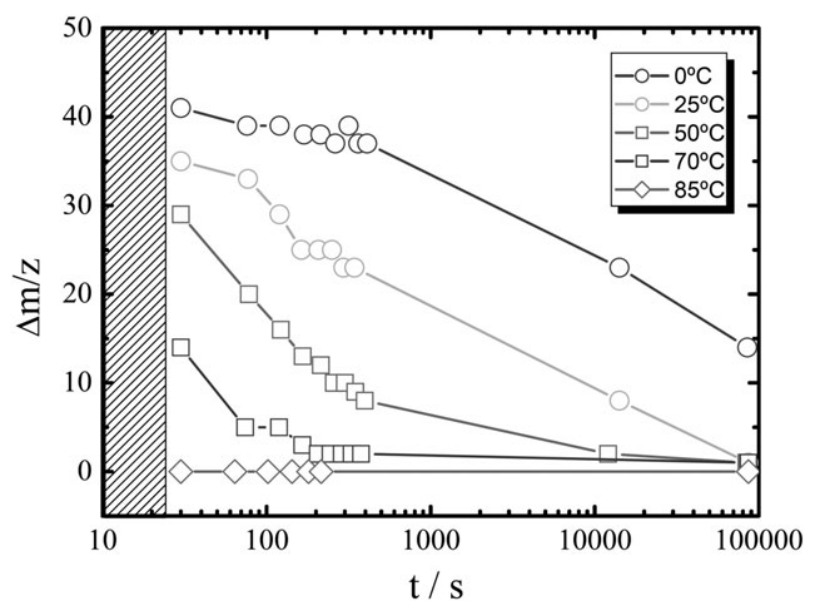

Fig. 19 H/D exchange kinetics of lysozyme as a function of time and temperature. Note, the first data point has been taken at $t \approx 25 \mathrm{~s}$. 
3.2.3 Oxidation of biomolecules. The role of free radicals in human health disorders has been progressively more studied in recent years. ${ }^{112}$ Nowadays it has been established that the oxidative damage caused by free radicals on proteins and other biological molecules is involved in the development of, for example, cardiovascular diseases, cancer, and Alzheimer disease. ${ }^{113,114}$ Different reactive oxygen species (ROS) such as $\mathrm{H}_{2} \mathrm{O}_{2}$ are biologically formed in large quantities in cells, as a result of regular metabolism. $\mathrm{H}_{2} \mathrm{O}_{2}$ decomposes and forms two very reactive $\mathrm{OH}$ radicals by the Fenton reaction or by the Haber-Weiss recombination. There is ample evidence to support the notion that the most important mechanism of oxidative damage to proteins is metal-catalyzed oxidation (MCO). The reaction of the hydroxyl radical $(\mathrm{OH})$ with biological macromolecules has been an area of extensive study. The reactions of $\mathrm{OH}$ with nucleic acids have also been used to map solvent-accessible surfaces. $\mathrm{OH}$ reactions with amino acids, peptides, and proteins are also of interest and the relative reactivity of the amino acids has been established by determining the second-order rate constants for the reaction of the $\mathrm{OH}$ with amino-acids using pulse radiolysis. ${ }^{115}$ However, determining the sites of reaction of $\mathrm{OH}$ has been more difficult. NMR, EPR radical trapping, and mass spectral characterization of the different $\mathrm{OH}$-induced products of amino-acids generated in aerobic aqueous solution support the conclusion that side chain reactions are the most prominent at physiological $\mathrm{pH}$. In the presence of oxygen, the aromatic and sulfur containing side chains are the most prominent sites of oxidation, with $\mathrm{O}$-atom incorporation being the main reaction. ${ }^{116}$

Beyond the investigation of oxidative stress in vivo protein foot-printing utilizing hydroxyl radicals coupled with mass spectrometry has become a powerful technique for mapping the solvent accessible surface of proteins and examining protein-protein interactions in solution. ${ }^{117}$ The hydroxyl radicals generated through the above methods can efficiently oxidize amino-acids on or near the surface of proteins in direct relation to their reactivity and their solvent accessibility. In particular, the solvent-accessible side chains of cysteine, methionine, phenylalanine, tyrosine, tryptophan, histidine, proline, and leucine provide convenient and useful probes for protein foot-printing. Since these residues cover $25-30 \%$ of the sequence of a typical protein, many sites throughout a protein's structure are potential probes for the method. Hydroxyl radical mediated foot-printing mass spectrometry (MS) is very similar to deuterium exchange MS methods except that the side chains instead of backbone positions are probed.

Amino-acids are the main building blocks of living systems, being the principal components of all natural peptides and proteins. In order to demonstrate the power of time-resolved IR-FL-MALDI-MS for the investigation of oxidation of biomolecules by $\mathrm{OH}$ radicals we have studied the reaction of $\mathrm{OH}$ and arginine. ${ }^{118}$ The reactive $\mathrm{OH}$ in aqueous solution was generated via Fenton's reaction, i.e., the reaction of $\mathrm{Fe}^{2+}$ salt and $\mathrm{H}_{2} \mathrm{O}_{2}$. The reaction was initiated by mixing the $\mathrm{Fe}^{2+} / \mathrm{H}_{2} \mathrm{O}_{2}$ with the arginine and injected in the source of the liquid beam MS. A characteristic experiment is displayed in Fig. 20a. As can be seen, after adding $\mathrm{Fe}^{2+} / \mathrm{H}_{2} \mathrm{O}_{2}(1 \mathrm{mM})$ the parent amino-acid $(0.1 \mathrm{mM})$ peak height at mass $175 \mathrm{Da}$ $\left(\mathrm{M}+\mathrm{H}^{+}\right)$decays within an hour to $1 / \mathrm{e}$ of its initial value and

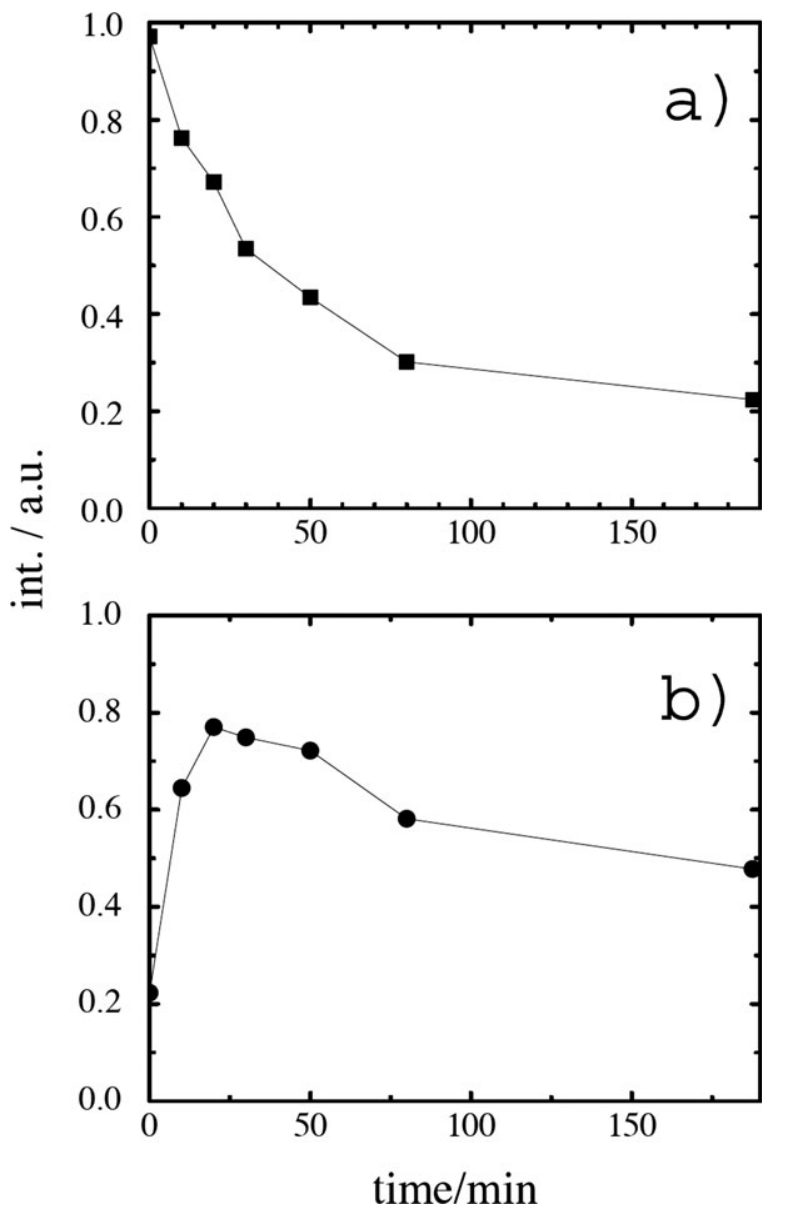

Fig. 20 Reaction of $\mathrm{OH}$ with arginine $(0.1 \mathrm{mM})$. $\mathrm{OH}$ from the reaction of $\mathrm{Fe}^{2+}+\mathrm{H}_{2} \mathrm{O}_{2}(1 \mathrm{mM})$. (a) Reaction and decomposition of the parent arginine (mass peak intensity of $\mathrm{M}+\mathrm{H}^{+}$at $175 \mathrm{Da}$ ). (b) Buildup of the guanidine product mass peak $\left(\mathrm{M}+\mathrm{H}^{+}\right.$at $\left.60 \mathrm{Da}\right)$ and its decay (pos. ion mode).

the decay slows down in turn after an hour. It is known that those amino-acids (e.g., arginine or histidine) are particularly reactive that may form a complex with metal salts such as $\mathrm{Fe}^{2+}$. At the site of complexation the metal ion reacts with $\mathrm{H}_{2} \mathrm{O}_{2}$ and forms $\mathrm{OH}$ which, in turn, is able to react in the near vicinity which is close to the complexed amino-acid. An intermediate detected in the course of the reaction with a mass of $60 \mathrm{Da}(\mathrm{M}$ $+\mathrm{H}^{+}$) results from the loss of the guanidinium group. ${ }^{115}$ The oxidation of arginine by metal ion-catalyzed oxidation systems has been reported before and the rate constants are in good agreement with the present experiments. ${ }^{115} \mathrm{~A}$ mechanism of arginine oxidation suggested in ref. 115 for metal-catalyzed oxidation explaining the formation of the -43 Da product with respect to the parent arginine and the appearance and decay of a 60 Da product (Fig. 20b) is given in Fig. 21. A hydroxyl radical abstracts a hydrogen from the $\delta$-carbon of the side chain and adds a hydroxyl group to form an intermediate oxidation product with +16 Da mass with respect to the parent arginine. The subsequent loss of the guanidine group from the $+16 \mathrm{Da}$ precursor oxidation product leads to $\gamma$-glutamyl semialdehyde. A similar oxidation of arginine is expected to take place under radiolytic conditions. ${ }^{115}$ 
<smiles>CN[C@@H](C[Te][Te]NC(=N)N)C(C)=O</smiles>
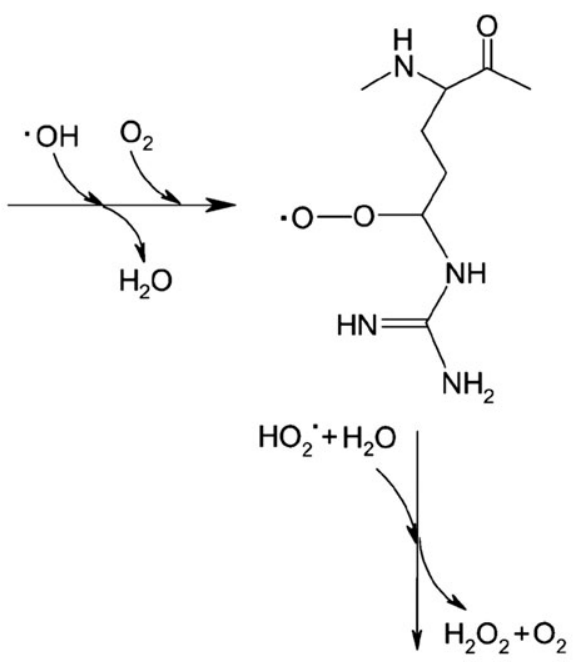<smiles>CNC(CC=O)C(C)=O</smiles>

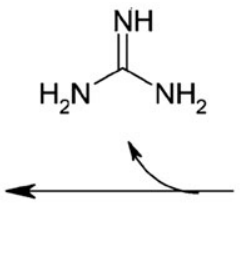

$M-43$

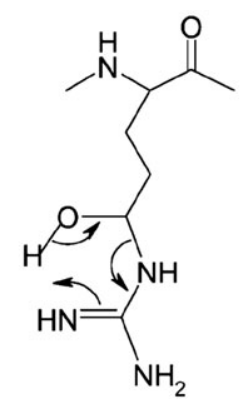

Fig. 21 Proposed mechanism ${ }^{115}$ for formation of -43 and $60 \mathrm{Da}$ oxidation product from arginine residue.

\subsection{Polymers}

Characterization of polymeric materials is vital for predicting and elucidating polymer properties and morphology. Characterization typically involves: (1) molecular mass analysis utilizing size exclusion chromatography, light scattering, osmometry, or viscometry; (2) sequence of repeat units utilizing NMR spectroscopy; (3) endgroup analysis utilizing titration, NMR spectroscopy, or FTIR spectroscopy; and (4) purity examination utilizing NMR spectroscopy, elemental analysis, and FTIR spectroscopy. ${ }^{119}$ Also, mass spectrometry (MS) has been used for the analysis of molar masses of molecules for quite some time. ${ }^{120,121}$ Most of the early problems associated with the application of MS to synthetic polymers have been overcome with the invention and introduction of soft ionization techniques. ${ }^{120,122,123}$ IR-FL-MALDI-MS was originally developed for the analysis of biomolecules but it has also emerged as a powerful technique for the characterization of synthetic polymers. ${ }^{32}$ It allows for the mass determination of large biomolecules, oligomers, ${ }^{61,124}$ and synthetic polymers of molar masses up to $2 \times 10^{5} \mathrm{Da}$ by direct desorption of ionic aggregates without fragmentation of the analyte molecules. At the same time the technique is also able to monitor the molar mass distribution of the macromolecules in cases of polydisperse polymers. ${ }^{32}$ In comparison with MALDI the discrimination of charged species in the spectrum, which manifests itself in a dominance of the low mass species in the spectrum, is by far not as pronounced for IR-FL-MALDI-MS. ${ }^{122}$ The Fig. a)

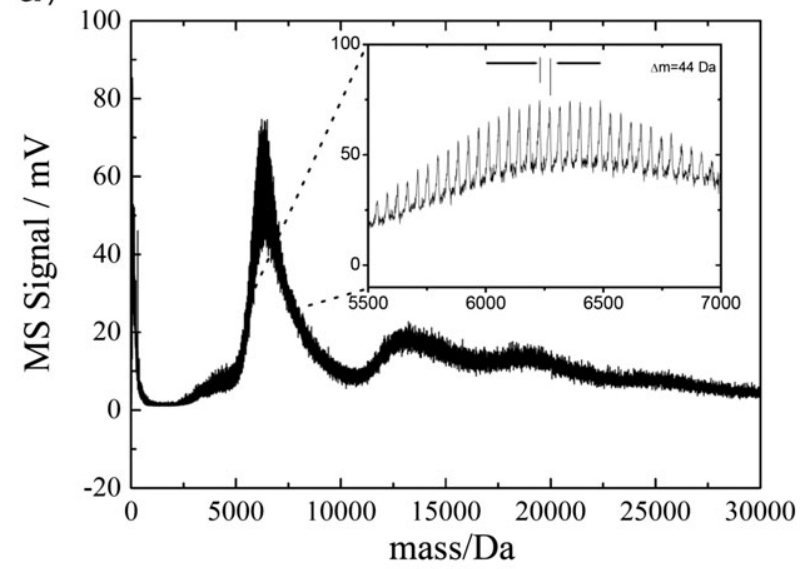

b)

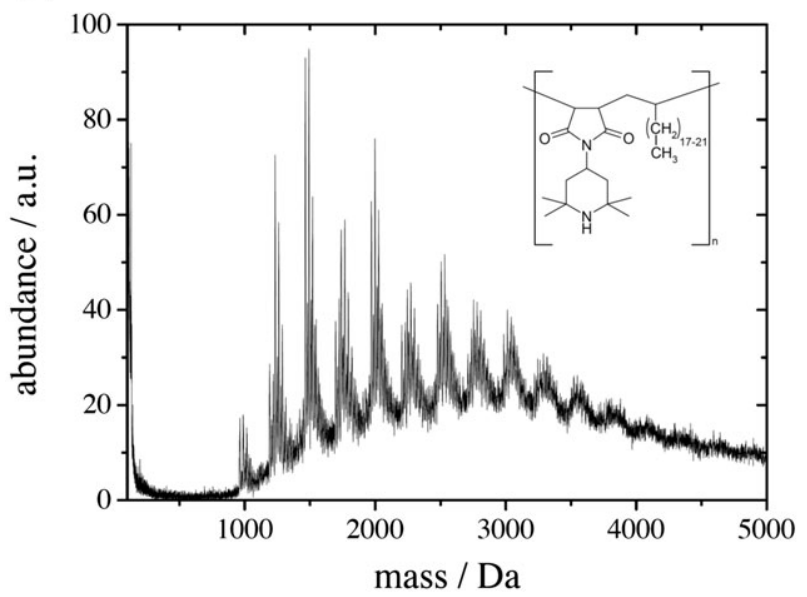

Fig. 22 (a) Mass spectrum of PEG 6000 (pos. ion mode, $\mathrm{pH}=7$ ) which displays the measured polymer mole mass distribution. Note, the seeming noise in the spectrum can be resolved and is due to different monomer units (see the inset). (b) Mass spectrum of a synthetic polymer (sterically hindered oligomeric amine, UVINUL ${ }^{\mathrm{TM}}$ ) used as a UV absorber, directly from a production batch (pos. ion mode, $\mathrm{pH}=3,0.1 \mathrm{M}$ acetic acid/water solution).

22a shows a polyethylene glycol (PEG6000) spectrum directly desorbed from the liquid water phase. It is interesting to see that the molar mass distribution peaks at $M=6000 \mathrm{Da}$ but extends beyond this value. Due to the nature of the liquid beam technique it is conceivable that this technique may be able to monitor polymerization reactions in the continuous mode. In Fig. 22b a spectrum of UVINUL - a sterically hindered oligomeric polyamine-from an industry polymerization batch is shown. Here the sampling was still discontinuous and the analysis was made off-line but it shows that the polymerization process can be analyzed and, in turn, controlled. For the particular polymer product it was important that the molar mass distribution does not extend towards low masses below $1000 \mathrm{Da}$, a direct application of product and quality control in industry.

\subsection{Multi-component analysis in complex fluids}

Quantitative measurements via mass spectrometry in general and liquid beam desorption MS in particular are possible if 
compound-to-compound response is uniform and varies linearly with concentration. Reduction or elimination of MS response resulting from the presence of another analyte is called "ion suppression" or a "matrix effect" when caused by another matrix component such as the solvent itself or the buffer. As demonstrated in recent articles, we have measured a large number of mixtures of proteins with varying concentrations with liquid beam desorption MS in order to investigate these effects. A representative example is shown is Fig. 23a. In this case the concentration of insulin was varied over 2-3
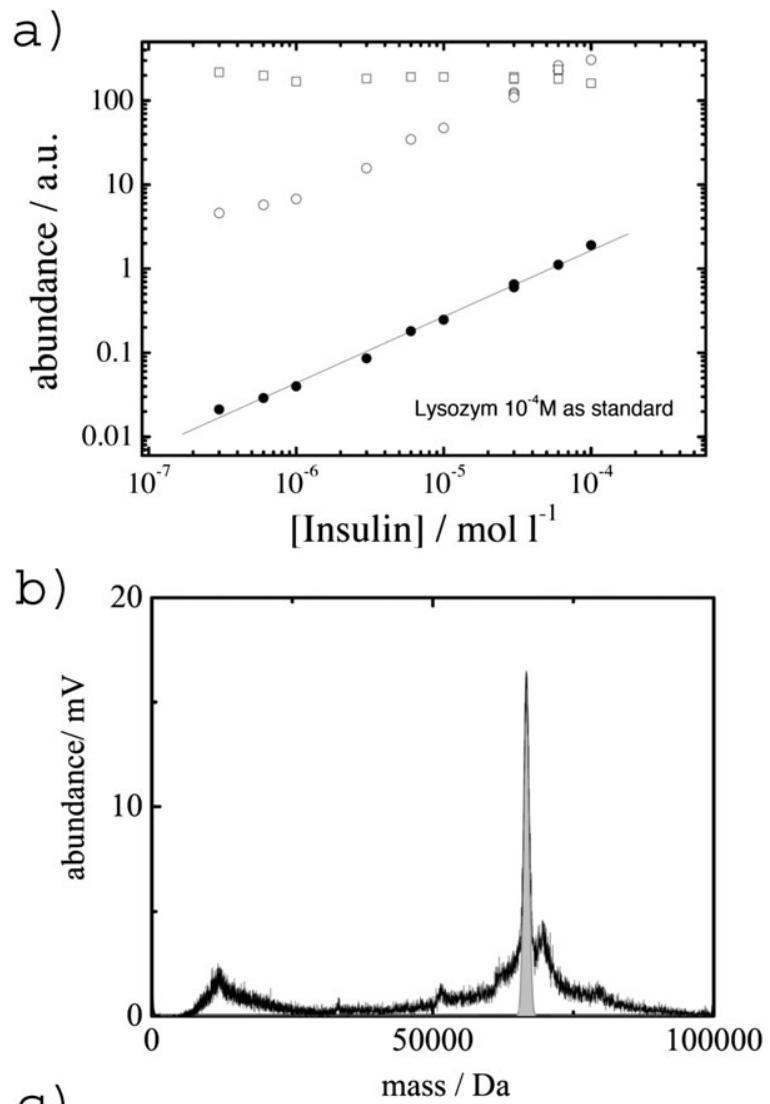

C)

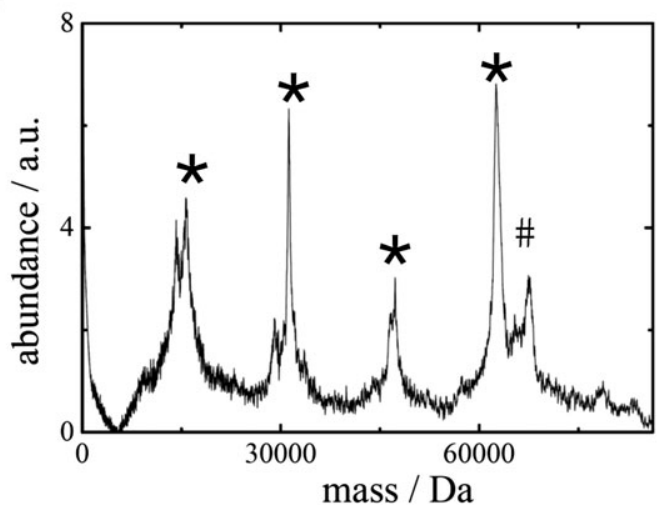

Fig. 23 (a) Mass peak intensities of insulin (filled circles, open circles) as a function of concentration with lysozyme (open squares) as a standard $(0.1 \mathrm{mM})$. For more details see the text. (b) Quantitative mass spectrum of BSA in bovine blood serum (diluted). (c) HSA (\#) and hemoglobin $\left(^{*}\right)$ mass spectrum from a fresh human blood sample (diluted) without centrifugation and special processing. All spectra recorded in positive ion mode (ammonium acetate buffer, $\mathrm{pH}=7$ ). orders of magnitude and the concentration of lysozyme was kept constant and was used as a standard. The filled circles in Fig. 23a represents the insulin signal (open circles) divided (normalized) by the lysozyme signal (open squares). Obviously, the interference between these proteins is small, although the slope of the insulin signal as a function of liquid concentration is somewhat lower than unity, possibly an as indication of a weak matrix effect in this system. A similar result has been found for other systems as well, ${ }^{31-33}$ putting us in a position to use a well detectable protein in solution as a standard. This feature may be particularly important for the accurate measurement of minor species concentrations in biomolecule kinetic studies.

In another recent study we have determined bovine serum albumin (BSA) directly and quantitatively in blood serum ${ }^{33}$ (see Fig. 23b). In this case the serum sample has been diluted by $1: 5$ (ammonium acetate buffer) and directly injected into the spectrometer. The quantitative result was compared with results from other standard techniques. ${ }^{125}$ In another experiment we have investigated human blood samples (Fig. 23c). Due to the high abundance of hemoglobin and human serum albumin in blood it was rather easy to detect both proteins quantitatively in blood. ${ }^{126}$ The estimated relative peak intensities correspond roughly to $40 \mathrm{~g} \mathrm{l}^{-1} \mathrm{HSA}$ and $120 \mathrm{~g} \mathrm{l}^{-1}$ hemoglobin, which is in the tolerance interval for these blood compounds for healthy adults. The interesting point in the latter case is not that the proteins can be detected. They are clearly the most abundant proteins in blood and not a minority species to be detected in the presence of large quantities of other proteins. The interesting point here is that the complex matrix (containing salt and proteins in large quantities) still enables the quantitative determination of these proteins. A second point is that the method is relatively soft, such that hemoglobin can be detected even in the tetrameric form, although fragmentation of the complex is visible under the present conditions. This result may be compared with the results from section 3.1 and Fig. 10a where pure (ammonium acetate buffered) hemoglobin in water solution has been investigated (showing less fragmentation). The present results are again a clear demonstration that the salt and matrix tolerance of the method is good and that the method may be soft and quantitative, even under "real world" conditions.

\subsection{A natural combination-all liquid state analysis via on-line coupling with HPLC}

From the very early stages of development of modern mass spectrometry, the value of its combination with chromatography has been recognized. The coupling of gas chromatography (GC) with MS was a natural evolution since they are both vapor phase techniques, and very quickly GC-MS has been accepted as a standard component of the organic analytical laboratory. It has taken considerably longer to achieve a satisfactory and all-purpose mode of HPLC-MS coupling. The difficulties with HPLC-MS were associated with the fact that vaporization of typically $1 \mathrm{~mL} \min ^{-1}$ from the HPLC translates into a vapor flow rate of approx. $500-1000 \mathrm{~mL}$ $\min ^{-1}$. Other difficulties were related to the eluent composition as a result of the frequent use of non-volatile modifiers, and 
the ionization of non-volatile and thermally labile analytes. However, during the past several years commercial interfaces have been developed (thermospray, electrospray, APCI interfaces, MALDI) which have led to a broad applicability of HPLC-MS in organic chemistry and biochemistry. ${ }^{57,127}$

A natural combination of two closely related techniques appears to be high pressure/performance liquid chromatography and IR-FL-MALDI-MS. Since the injection of a liquid microbeam into vacuum requires already a HPLC pump, the combination of both techniques appeared to be quite straightforward. An illustrative example is displayed in Fig. 24 for a separation of three cyclodextrins. Here the IR-FL-MALDI source of the mass spectrometer was directly interfaced and fed by the high-pressure exit of the commercial HPLC unit. In Fig. 24a the mass spectrum of a mixture (roughly of the same concentration, $\approx 0.1 \mathrm{mM}$ ) of $\alpha$-, $\beta$-, and $\gamma$-dextrins in a water methanol mixture $(4: 1)$ is shown. In this case the dextrins are desorbed as sodium adducts, $\mathrm{M}-\mathrm{Na}^{+}$. The cyclodextrins are structurally quite similar and pose a simple separation problem for a first proof-of-principle of the combination of IRFL-MALDI-MS and HPLC. For the separation a RP18 column in a KNAUER HPLC system (SMARTLINE ${ }^{\mathrm{TM}}$ ) equipped with a refractive index detector (RI) and a UV detector. The inset in Fig. 24b displays two separated peaks detected with the RI-detector. The Fig. 24b shows time dependent intensities of mass peaks after partial HPLC separation coupled on-line with IR-FL-MALDI-MS. In this experiment we worked with a RP18 column at $T=30{ }^{\circ} \mathrm{C}$ with a flow rate of $0.3 \mathrm{ml} \mathrm{min}{ }^{-1}$, and a sample loop of $20 \mu$ l. The mobile phase was $\mathrm{H}_{2} \mathrm{O}: \mathrm{MeOH}(4: 1)$. From an inspection of Fig. $24 \mathrm{~b}$ it is easy to see that the unresolved peaks in the HPLC separation detected with the RI detector can easily be mass resolved and quantitatively detected with LBD-MS in this case. In Fig. $24 \mathrm{c}$ we have checked the linear response of mass peaks on solution concentration after HPLC separation and LBD-MS for $\beta$ - and $\gamma$-cyclodextrin for various concentrations. The nearlinear response and the all-liquid state technology should be interesting for HPLC-MS coupling in the future even though several commercial systems exist. As a final point we note that other solvents with IR excitable bonds (alcohols) and solvent mixtures (dioxane, acetonitrile) and typical HPLC gradients (also time dependent gradients) - as long as the water content is above $20 \%$ - as not a problem. Even the presence of TFA was not limiting the performance. A development of the technique into nanoflow liquid chromatography/IR-FLMALDI-MS should be straightforward with a droplet or SCLICED version of IR-FL-MALDI-MS and is underway in the laboratory of the authors.

\subsection{Quantitative measurement of oxidation states in solution}

A technique that allows the transfer of ions from solution into the gas phase, from which the ions can be subjected to mass spectrometric analysis, is of great importance because nearly half of the chemical and biochemical processes involve ions in solution. Electrospray ionization is such a technique. ${ }^{128,129} \mathrm{It}$ affords ion transfer into the gas phase and detection of a wide variety of ions dissolved in a wide variety of solvents. When it comes to the quantifications of ions in solution it is important

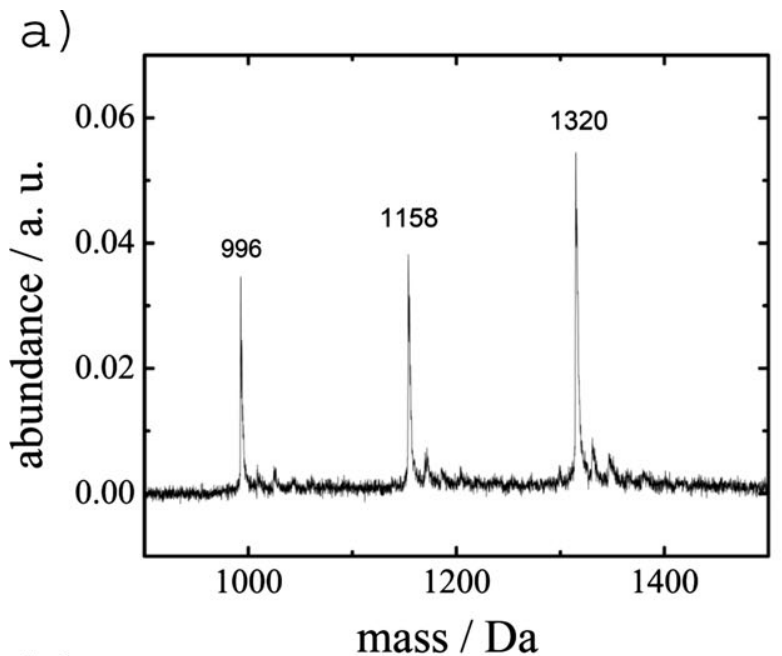

b)
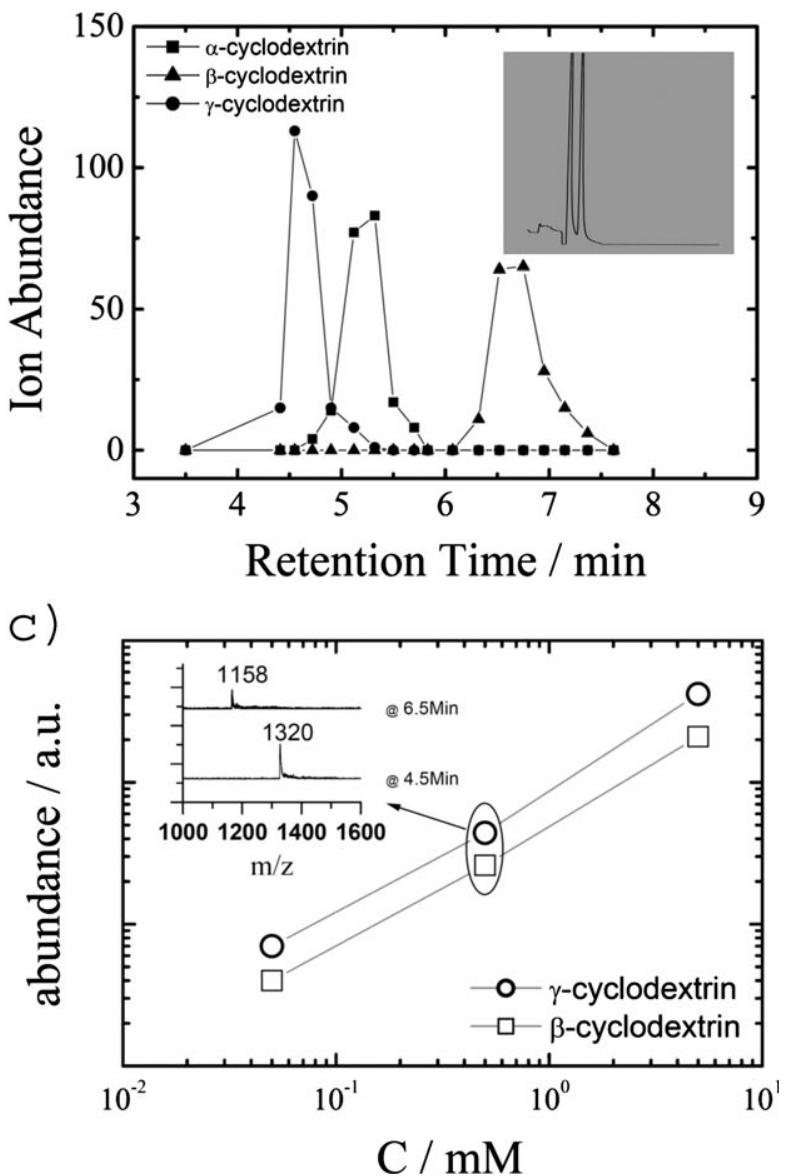

Fig. 24 (a) Spectrum of a mixture $(0.1 \mathrm{mM})$ of $\alpha-, \beta$-, and $\gamma$-cyclodextrins in water/methanol solution $(4: 1)$. (b) Time dependent intensities of mass peaks after partial HPLC separation $(\mathrm{pH}=7)$. Inset: signal of the refractive index detector. HPLC settings: column

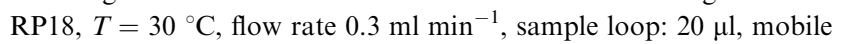
phase $\mathrm{H}_{2} \mathrm{O}: \mathrm{MeOH}(4: 1)$. (c) Linear response of mass peak signal after HPLC separation of $\beta$ - and $\gamma$-cyclodextrin. Spectra recorded in positive ion mode.

to (i) measure ions quantitatively in solution, and (ii) not to alter their oxidation state if the ions can be in several oxidation states. Although ESI has been used to monitor ion 
concentrations in environmental samples and salt solutions it is not without problems. The first problem arises from the nonlinear response of the technique, i.e., the nonlinearity of the gas phase signal upon the solution concentration. The other problem is that the electrospray technique is intrinsically able to modify charge states of ions in solution. ${ }^{128}$ Assuming that the charge separation in ESI is electrophoretic, at a steady operation of the ESI, positive droplet emission will continuously carry off positive droplet or ions. The requirement for charge balance in such a continuous-electric-current device, together with the fact that only electrons can flow through the metal wire supplying the electric potential to the electrodes, leads to the supposition that the ESI process must include an electrochemical conversion of ions to electrons. In other words, the ES device may be viewed as a special type of electrolytic cell. ${ }^{128}$ This feature is important when metal ions such as $\mathrm{Cr}$ or $\mathrm{Fe}$ in different oxidation states are present together in the sample solution and if one wishes to measure both quantitatively. Up to now a special analytical problem is to measure species in different oxidation states simultaneously with a mass spectrometric technique without changing oxidation numbers (if the change of oxidation state by an exchange redox-reaction can be excluded). Although metal ion and oxidation number speciation has been possible with UVdesorption aerosol mass spectrometry ${ }^{130}$ some time ago, quantitative measurements are still a general and major challenge. By its nature, IR-FL-MALDI-MS does not change oxidation numbers of ions in solution (at least we have no indications that redox reactions such as those reported in ref. 61 play a significant role in our conditions) and it is able to measure ion concentrations and oxidation states quantitatively (if other fast redox-reactions in solution can be excluded). This was the motivation to apply the technique to the quantitative detection of $\mathrm{Fe}^{2+}$ and $\mathrm{Fe}^{3+}$ in aqueous solution. Salts in different oxidations states often have different solubility. In order to circumvent this problem we have investigated the metal-ligand complexes $\mathrm{K}_{4}\left[\mathrm{Fe}(\mathrm{CN})_{6}\right]$ and $\mathrm{K}_{3}\left[\mathrm{Fe}(\mathrm{CN})_{6}\right]$ known as the yellow and red prussiates of potash. ${ }^{131}$ Their exchange rates are fortunately slow enough such that the initial concentrations can be measured without perturbation. Fig. 25a displays a mass spectrum of $\mathrm{K}_{4}\left[\mathrm{Fe}(\mathrm{CN})_{6}\right]^{+}$and $\mathrm{K}_{5}\left[\mathrm{Fe}(\mathrm{CN})_{6}\right]^{+}$showing a typical isotope pattern. Fig. 25b shows the concentration dependence of the $\mathrm{Fe}^{3+}$ salt ion signal keeping the $\mathrm{Fe}^{2+}$ salt concentration constant (plotted is the ratio of the $\mathrm{K}_{4}\left[\mathrm{Fe}(\mathrm{CN})_{6}\right]^{+}$and $\mathrm{K}_{5}\left[\mathrm{Fe}(\mathrm{CN})_{6}\right]^{+}$mass peaks). A near linear response of the $\mathrm{Fe}^{3+}$ complex mass peak as a function of concentration was observed when the concentration of the $\mathrm{Fe}^{2+}$ complex was kept at $1 \mathrm{mM}$. These experimental results shown impressively that the salt complexes in aqueous solution can be measured quantitatively and that the oxidation number is not significantly influenced or changed by the technique. $\mathrm{Fe}^{2+} / \mathrm{Fe}^{3+}$ conversion in solution is in this case slow, probably due to complexation. It is also interesting to see that due to the relatively high cation concentration $\left(\mathrm{K}^{+}\right)$the complexes are desorbed unfragmented as $\mathrm{K}_{4}\left[\mathrm{Fe}(\mathrm{CN})_{6}\right]^{+}$and $\mathrm{K}_{5}\left[\mathrm{Fe}(\mathrm{CN})_{6}\right]^{+}$ complexes and not as protonated or deprotonated complexes or fragments. Recent studies in our lab to be published elsewhere have shown that the concept of quantitatively measur-
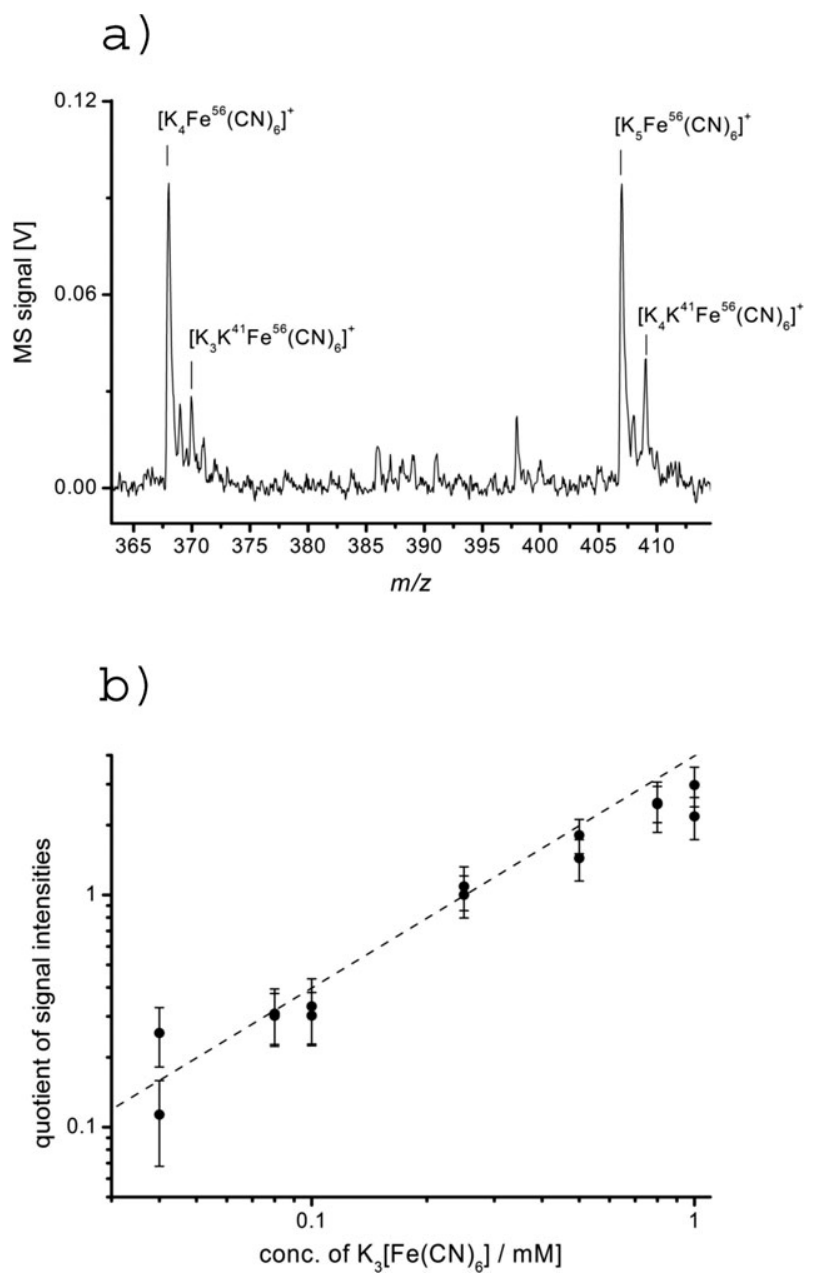

Fig. 25 (a) Mass spectrum of $\mathrm{K}_{4}\left[\mathrm{Fe}(\mathrm{CN})_{6}\right]^{+}$and $\mathrm{K}_{5}\left[\mathrm{Fe}(\mathrm{CN})_{6}\right]^{+}$ complexes desorbed from liquid water displaying a typical isotope pattern; (b) concentration dependence of the $\mathrm{Fe}^{3+}$ salt ion signal keeping the $\mathrm{Fe}^{2+}$ salt concentration constant (plotted is the ratio of the $\mathrm{K}_{4}\left[\mathrm{Fe}(\mathrm{CN})_{6}\right]^{+}$and $\mathrm{K}_{5}\left[\mathrm{Fe}(\mathrm{CN})_{6}\right]^{+}$mass peaks). A near-linear response of the $\mathrm{Fe}^{3+}$ complex mass peak as a function of concentration was observed when the concentration of the $\mathrm{Fe}^{2+}$ complex was kept at $1 \mathrm{mM}$. Spectra recorded in positive ion mode $(\mathrm{pH}=7)$.

ing oxidation states is applicable in general (even in timeresolved kinetic experiments) and not limited to the examples (complexes) and static concentrations shown here. ${ }^{131}$

\section{Summary, conclusions and outlook}

In the present feature article, some applications, the physical background and characteristic features of the IR-FL-MALDIMS approach have been highlighted (in comparison with more established techniques). The examples show that the technique is steadily maturing since it has first been reported, ${ }^{19}$ and it is on the way to become a competitive alternative source concept to the established MALDI and ESI techniques. Due to its features, such as the linear response, it appears well suited for time resolved kinetic measurements of biomolecule transformations. At the same time coupling to other separation techniques such as high performance liquid chromatography has been demonstrated to be straightforward and powerful, 
due to the all-liquid-state technology. Through the introduction of liquid beam segment desorption and droplet desorption the problem of too high analyte consumption appears to be largely resolved or resolvable in the near future. The technique has been applied to a broad range of problems related to molecular analysis and biokinetics of supramolecular systems. Some of them are highlighted in the present feature article. An important feature to point out here is that IR-FL-MALDIMS has become a powerful tool for solution spectroscopy, enabling the determination of true rate constants of biomolecule transformations in solution. These features could also help to make the technique useful in the field of metabolomics in the future. The SCLICED technique may be of particular interest for ultra-high throughput screening studies with a potential that has not come to its limits yet. Among the analytical techniques currently used in the characterization of synthetic polymers, mass spectrometry is of increasing importance. Also in this field IR-FL-MALDI-MS (also from liquid mixtures containing at least some water) appears to be very promising. This feature, among the others discussed in this overview, will open up interesting new possibilities in the future in the field of (online) polymer analysis and quality control. The analytic power in analyzing complex fluids or mixtures online may also provide interesting new opportunities for biotechnology, e.g., the application of (time resolved) liquid beam desorption mass spectrometry for monitoring bioreactors.

In summary, after nearly a decade of research on liquid beam desorption mass spectrometry it is closing up to the established techniques like MALDI and ESI and has now clearly matured to a powerful source concept for mass spectrometry that enables the direct and quantitative mass analysis of supramolecular species from liquid samples such as water, alcohols and solvent mixtures (e.g., acetonitrile) containing water or alcohol (as long as their content is above $20-30 \%$ ). The characteristic and main advantage-beyond its feature that it is an interesting (and maybe complementary) source concept for mass spectrometry - is that the technique makes mass spectrometry a powerful and quantitative liquid state spectroscopy with important and powerful features such as high sensitivity, selectivity, good time resolution, and a multiplex advantage.

\section{Acknowledgements}

The work described and highlighted here would have not been possible without the contributions of other past and present co-workers and group members (A. Boegehold, A. Beinsen, V. Suarez, Dr Manfred Faubel, Dr B. Stasicki, Dr F. Gessler, Dr J. Niemeyer, E. Vöhringer-Martinez). Support from the German Science Foundation (DFG) within the Graduate School Program 782, the Ministry of Trade and Commerce of Lower Saxony ("Development of Multidimensional Analytical Techniques for Proteomics", Verbund Projekt:80008452), and the Max-Planck-Society are gratefully acknowledged. In the project we have also significantly benefited from the stimulus of collaborations and discussions with Dr M. Faubel, E. Vöhringer-Martinez, Dr J. Niemeyer, Dr E. Mandelkow, Prof. Dr H. Grubmüller, Prof. Dr Ch. Griesinger, as well as Dr T.
Jovin. Discussions of some aspects of the presented work with Prof. Dr B. Brutschy and Prof. Dr F. Hillenkamp were also valuable for the work reviewed in this article. Finally, our thanks go to A. Beinsen, V. Suarez, and A. Boegehold for their help in the preparation of some of the figures and to the reviewers for helpful comments.

\section{References}

1 M. L. Vestal, Chem. Rev., 2001, 101, 361.

2 R. J. Levis, Annu. Rev. Phys. Chem., 1994, 45, 483.

3 P. Chen, Angew. Chem., 2003, 115, 2938.

4 K. M. Downnard, J. Mass Spectrom., 2000, 35, 493.

5 T. Tanaka, M. Waki, Y. Ido, Y. Akita, Y. Yoshida and T. Yoshida, Rap. Commun. Mass Spectrom., 1988, 2, 151.

6 M. Karas and F. Hillenkamp, Anal. Chem., 1988, 60, 2299.

7 J. B. Fenn, M. Mann, C.-K. Meng, S.-F. Wong and G. M. Whitehouse, Science, 1989, 246, 64.

8 K. C. Chan, D. A. Lucas, D. Hise, C. F. Schaefer, Z. Xiao and G. M. Janini, Clin. Proteom., 2004, 1(2), 101.

9 M. Mann, Nature, 2002, 418, 731.

10 J. Wei, J. M. Buriak and G. Siuzdak, Nature, 1999, 399, 243.

11 R. G. Cooks, Z. Ouyang, Z. Takats and J. M. Wiseman, Science, 2006, 311, 1566.

12 C. R. Blakeley, M. J. McAdams and M. L. Vestal, J. Chromatogr., $A, 1978, \mathbf{1 5 8}, 261$.

13 F. Hillenkamp, M. Karas and S. Berkenkamp, Proc. 43th Annual ASMS Conference on Mass Spectrometry and Allied Topics, 1995, 21-26 May, Atlanta, GA, USA, p. 357.

14 S. Berkenkamp, C. Menzel, M. Karas and F. Hillenkamp, Rap. Commun. Mass Spectrom., 1997, 11, 1399.

15 S. Berkenkamp, M. Karas and F. Hillenkamp, Proc. Natl. Acad. Sci. U. S. A., 1996, 93, 7003.

16 M. Faubel and T. Kisters, Nature, 1989, 339, 527.

17 M. Faubel, S. Schlemmer and J. P. Toennies, Z. Phys. D, 1988, 10, 269.

18 M. Faubel and B. Steiner, Ber. Bunsen-Ges. Phys. Chem., 1992, 96, 1167.

19 W. Kleinekofort, J. Avdiev and B. Brutschy, Int. J. Mass Spectrom. Ion Processes, 1996, 152, 135.

20 A. Wattenberg, H.-D. Barth and B. Brutschy, J. Mass Spectrom., 1997, 32, 1350.

21 A. Charvat, E. Lugovoj, M. Faubel and B. Abel, Eur. Phys. J. D, $2002,20,573$.

22 N. Morgner, H.-D. Barth and B. Brutschy, Aust. J. Chem., 2006, 59, 109.

23 W. Kleinekofort, A. Pfenninger, T. Plomer, C. Griesinger and B. Brutschy, Int. J. Mass Spectrom. Ion Processes, 1996, 156, 195.

24 W. Kleinekofort, M. Schweitzer, J. W. Engels and B. Brutschy, Int. J. Mass Spectrom. Ion Processes, 1997, 163, $1 \mathrm{~L}$.

25 F. Sobott, W. Kleinekofort and B. Brutschy, Anal. Chem., 1997, 69, 3587.

26 F. Sobott, S. A. Schunk, F. Schüth and B. Brutschy, Chem.-Eur. J., 1998, 4, 2353.

27 F. Sobott, A. Wattenberg, H.-D. Barth and B. Brutschy, Int. J. Mass Spectrom., 1999, 185-187, 271.

28 F. Sobott, A. Wattenberg, W. Kleinekofort, A. Pfenninger and B. Brutschy, Fresenius' J. Anal. Chem., 1998, 360, 745.

29 A. Wattenberg, F. Sobott, H.-D. Barth and B. Brutschy, Int. J. Mass Spectrom., 2000, 203, 49.

30 A. Wattenberg, F. Sobott and B. Brutschy, Rap. Commun. Mass Spectrom., 2000, 14, 859.

31 B. Abel, A. Charvat, U. Diederichsen, M. Faubel, B. Girmann, J. Niemeyer and A. Zeeck, Int. J. Mass Spectrom., 2005, 243, 177.

32 A. Charvat, A. Boegehold and B. Abel, Aust. J. Chem., 2006, 59, 81.

33 A. Charvat, F. Gessler, J. Niemeyer, A. Boegehold and B. Abel, Anal. Lett., 2006, 39, 2191.

34 A. Charvat, E. Lugovoj, M. Faubel and B. Abel, Rev. Sci. Instrum., 2004, 75, 1209.

35 A. Charvat, B. Stasicki and B. Abel, J. Phys. Chem. A, 2006, 110, 3297.

36 T. Spangenberg and B. Abel, Photonik, 2004, 6, 48. 
37 B. Stasicki, A. Charvat, M. Faubel and B. Abel, SPIE proceedings of the 26th International conference on high-speed photography and photonics, ed. Dennis L. Paisley, Stuart Kleinfelder, Donald R. Snyder and Brian J. Thompson, 2005, 5580, p. 335.

38 T. Kondow and F. Mafune, Annu. Rev. Phys. Chem., 2000, 51, 731.

39 W. L. Holstein, L. M. Dobeck, D. E. Otten, G. F. Metha and M. A. Buntine, Aust. J. Chem., 2003, 56, 481.

40 W. L. Holstein, M. R. Hammer, G. F. Metha and M. A. Buntine, Int. J. Mass Spectrom., 2001, 207, 1.

41 N. Horimoto, J. Kohno, F. Mafune and T. Kondow, J. Phys. Chem. A, 1999, 103, 9569.

42 N. Horimoto, F. Mafune and T. Kondow, J. Phys. Chem., 1996, 100, 10046.

43 N. Horimoto, F. Mafune and T. Kondow, J. Phys. Chem. B, 1999, 103, 9540.

44 J. Kohno, F. Mafune and T. Kondow, J. Am. Chem. Soc., 1994, 116, 9801.

45 J. Kohno, F. Mafune and T. Kondow, J. Phys. Chem. A, 2000, 104, 243.

46 J. Kohno, F. Mafune and T. Kondow, J. Phys. Chem. A, 2001, $\mathbf{1 0 5}, 8939$.

47 J. Kohno, F. Mafune and T. Kondow, Chem. Lett., 2002, xx, 562.

48 J. Kohno, F. Mafune and T. Kondow, J. Phys. Chem. A, 2004, 108, 971

49 O. J. Maselli, S. R. Gascooke, S. L. Kobelt, G. F. Metha and M. A. Buntine, Aust. J. Chem., 2006, 59, 104.

50 Y. Takeda, T. Kondow and F. Mafune, Nucleosides, Nucleotides Nucleic Acids, 2005, 24, 1215.

51 J. M. Daniel, V. V. Laiko, V. M. Doroshenko and R. Zenobi, Anal. Bioanal. Chem., 2005, 383, 895.

52 N. Mano and J. Goto, Anal. Sci., 2003, 10, 3.

53 D. O. O'Connor, D. E. Clarke, D. Morrison and A. P. Watt, J. Med. Chem., 2002, 37, 1385.

$54 \mathrm{M}$. Faubel, in 'Photoelectron spectroscopy at liquid surfaces', Advanced Series in Physical Chemistry - vol. 10A (Photoionization and Photodetachment Part 1), ed. C. Y. Ng, World Scientific, New York, 2000, p. 634.

55 E. Woods, Y. Dessiaterik, R. E. Miller and T. Baer, J. Phys. Chem. A, 2001, 105, 8273 .

56 O. Link, E. Lugovoj, M. Faubel, E. Vöhringer-Martinez, H. Grubmüller and B. Abel, 2007, submitted for publication.

57 K. K. Murray, Mass Spectrom. Rev., 1997, 16, 283.

58 E. Woods, R. E. Miller and T. Baer, J. Phys. Chem. A, 2003, 107, 2119

59 E. Woods, G. D. Smith, R. E. Miller and T. Baer, Anal. Chem., 2002, 74, 1642.

60 S. Woutersen, U. Emmerichs and H. J. Bakker, Science, 1997, 278, 658 .

61 F. Sobott, PhD Thesis, Johann-Wolfgang Goethe University, Frankfurt, 2000, published 2001 at dissertation.de, ISBN: 3898250296.

62 K. K. Murray, T. M. Lewis, M. D. Beeson and D. Russel, Anal. Chem., 1994, 66, 1601.

63 T. Nordmeyer and K. A. Prather, Anal. Chem., 1994, 66, 3540.

64 K. A. Prather, T. Nordmeyer and K. Salt, Anal. Chem., 1994, 66, 1403.

65 P. T. Steele, H. T. Tobias, D. P. Ferguson, M. E. Pitesky, J. M. Horn, G. A. Czerwieniec, S. C. Russel, C. B. Lebrilla, E. E. Gard and M. Frank, Anal. Chem., 2003, 75, 5480.

66 M. Karas and R. Krueger, Chem. Rev., 2003, 103, 427.

67 R. Zenobi and R. Knochenmuss, Mass Spectrom. Rev., 1998, 17, 337.

68 S. Berkenkamp, C. Menzel, M. Karas and F. Hillenkamp, Rap. Commun. Mass Spectrom., 1997, 11, 1399.

69 K. Dreisewerd, S. Berkenkamp, A. Leisner, A. Rohlfing and C. Menzel, Int. J. Mass Spectrom., 2003, 226, 189.

70 S. Berkenkamp, M. Karas and F. Hillenkamp, Proc. Natl. Acad. Sci. U. S. A., 1996, 93, 7003.

71 J. A. Loo, Int. J. Mass Spectrom., 2000, 200, 175.

72 P. G. Debenedetti, Metastable Liquids: Concepts and Principles, Princeton University Press, Princeton, NJ, 1996.

73 E. Vöhringer-Martinez, F. Wiederschein, A. Beinsen, A. Charvat, B. Abel and H. Grubmüller, Phys. Rev. Lett., 2007, submitted.

74 P. Kebarle, J. Mass Spectrom., 2000, 35, 804.
75 H. L. Friedman, Ionic solution theory, Wiley, New York, 1962.

76 G. Fermi, M. F. Perutz, B. Shaanan and R. Fourme, J. Mol. Biol., 1984, 175, 159.

77 W. P. Griffith and I. A. Kaltashov, Biochemistry, 2003, 42, 10024.

78 W. Zhang, S. Niu and B. T. Chait, J. Am. Soc. Mass Spectrom., $1998,9,879$

79 M. O. Glocker, S. H. J. Bauer, J. Kast, J. Volz and M. Przybylski, J. Mass Spectrom., 1996, 31, 1221.

80 R. P. Hearn, F. M. Richards, J. M. Sturtevant and G. D. Watt, Biochemistry, 1971, 10, 806.

81 R. P. Hearn, F. M. Richards, J. M. Sturtevant and G. D. Watt, Biochemistry, 1971, 10, 806.

82 F. M. Richards and A. D. Logue, J. Biol. Chem., 1962, 237, 3693.

83 A. A. Schreier, Biochemistry, 1977, 16, 4203.

84 A. A. Schreier and R. L. Baldwin, J. Mol. Biol., 1976, 105, 409.

85 A. A. Schreier and R. L. Baldwin, Biochemistry, 1977, 16, 4203.

86 I. A. Kaltashov and S. J. Eyles, Mass. Spectrm. Rev., 2002, 21, 37.

87 L. Konermann and D. A. Simmons, Mass Spectrom. Rev., 2003, 22, 1.

88 M. Brivio, R. H. Fokkens, W. Verboom, D. N. Reinhoudt, N. R. Tas, M. Goedbloed and A. van den Berg, Anal. Chem., 2002, 74, 3972.

89 C. T. Houston, W. P. Taylor, T. S. Widlanski and J. P. Reilly, Anal. Chem., 2000, 72, 3311.

90 I. A. Kaltashov and S. J. Eyles, in Mass Spectrometry in biophysics: Conformation and dynamics of biomolecules, ed. D. M. Desiderio, Wiley- Interscience Series in Mass Spectrometry, Wiley-Interscience, New York, 2005.

91 C. M. Dobson, Nature, 2003, 426, 884.

92 J. L. Jimenez, E. J. Nettleton, M. Bouchard, C. V. Robinson, C. M. Dobson and H. R. Saibil, Proc. Natl. Acad. Sci. U. S. A., 2002, 99, 9196.

93 R. Jansen, W. Dzwolak and R. Winter, Biophys. J., 2005, 88, 1344.

94 J. Brange, L. Andersen, E. Laursen, G. Mayn and E. Rasmussen, J. Pharm. Sci., 1997, 86, 517.

95 B. Abel, A. Bögehold, A. Charvat, U. Diederichsen, B. Herzog, T. Jovin, A. Ray, T. Salditt and H. Schollmeyer, Acc. Chem. Res., 2007, submitted.

96 J. W. Kelly, Nat. Struct. Biol., 2000, 7, 824.

97 T. R. Serio, A. G. Cashikar, A. S. Kowal, G. J. Sawicki, J. J. Moslehi, L. Serpell, M. F. Arnsdorf and S. Lindquist, Science, 2000, 289, 1317.

98 P. Friedhoff, M. van Bergen, E.-M. Mandelkov and E. Mandelkov, Biochim. Biophys. Acta, 2000, 1502, 122.

99 V. Makrides, M. R. Massie, S. C. Feistein and J. Lew, Proc. Natl. Acad. Sci. U. S. A., 2004, 101, 6746.

100 P. Friedhoff, M. von Bergen, E.-M. Mandelkov, P. Davies and E. Mandelkov, Proc. Natl. Acad. Sci. U. S. A., 1998, 95, 15712.

101 M. van Bergen, S. Barghorn, L. Li, A. Marx, J. Biernat, E.-M. Mandelkov and E. Mandelkov, J. Mol. Biol. Chem., 2001, 276, 48165.

102 B. Abel, A. Boegehold, A. Charvat, M.-K. Cho, C. Griesinger, E. M. Mandelkow, M. Mukrasch and M. Zweckstaetter, J. Am. Soc. Mass Spectrom., 2007, in press.

103 M. R. Nilsson, Methods, 2004, 34, 151.

104 S. W. Englander and L. Mayne, Annu. Rev. Biophys. Biomol. Struct., 1992, 21, 243

105 S. Segawa, Y. Husimi and A. Wada, Biopolymers, 1973, 12, 2521.

106 C. K. Woodward and B. D. Hilton, Ann. Rev. Biophys. Bioeng., $1979,8,99$.

107 S. W. Englander, N. W. Downer and H. Teitelbaum, Annu. Rev. Biochem., 1972, 41, 903.

108 E. W. Chung, E. J. Nettleton, C. J. Morgan, M. Griß, A. Miranker and S. E. Radford, Protein Sci., 1997, 6, 1316.

109 A. Hvidt and S. O. Nielsen, Adv. Protein Chem., 1966, 21, 287.

110 S. E. Radford, C. M. Dobson and P. A. Evans, Nature, 1992, 358, 302 .

111 V. Suarez-Ulloa, Masters Thesis, Isotope exchange of lysozyme observed by microbeam desorption mass spectrometry, University of Göttingen, 2006.

112 J.-W. Lee and J. D. Helmann, Nature, 2006, 440, 363.

113 D. J. Walther, J.-U. Peter and M. Bader, Cancer, 2002, 94, 3135

114 B. J. Tabner, O. M. A. El-Agnaf, M. J. German, N. J. Fullwood and D. Allsop, Biochem. Soc. Trans., 2005, 33, 1082. 
115 X. Guozhong, K. Takamoto and M. Chance, Anal. Chem., 2003, $\mathbf{7 5}, 6995$

116 B. N. Nukuma, M. B. Goshe and V. E. Anderson, J. Am. Chem. Soc., 2001, 123, 1208.

117 S. D. Maleknia et al., Anal. Biochem., 2001, 289, 103.

118 A. Beinsen, Diploma thesis, University of Göttingen, 2007.

119 M. P. Stevens, Polymer chemistry: An Introduction, Oxford University Press, Oxford, 1999.

120 C. N. McEven and P. M. Peacock, Anal. Chem., 2002, 74, 2743.

121 R. F. Rabeck and J. F. Rabeck, Lasers in Polymer Science and Technology Applications, CRC Press, Boca Raton, FL, 1989.

122 M. W. F. Nielsen, Mass Spectrom. Rev., 1999, 18, 309.

123 G. Montaudo and R. P. Lattimer, in Mass Spectrometry of Polymers, ed. G. Montaudo and R. P. Lattimer, CRC Press, Boca Raton, FL, 2001.
124 P. Bussian, F. Sobott, B. Brutschy, W. Schrader and F. Schüth, Angew. Chem., Int. Ed., 2000, 39, 3901.

125 K. Adachi, H. Kawano, K. Tsuno, Y. Nomura, N. Katsura, A. Arikawa, A. Tsuji and T. Onimaru, J. Vet. Med. Sci., 1997, 59, 873.

126 A. Boegehold, A. Charvat and B. Abel, unpublished results, 2006.

127 S. Kromidas, Practical problem solving in HPLC, Wiley, Weinheim, 2004.

128 G. J. Van Berkel, in Electrospray Ionization Mass Spectrometry: Fundamentals, Instrumentation and Applications, ed. R. B. Cole, John Wiley, New York, 1997.

129 V. B. Di Marco and G. G. Bombi, Mass Spectrom. Rev., 2006, 25, 347.

130 K. Neubauer, M. V. Johnston and A. S. Wexler, Int. J. Mass Spectrom. Ion Processes, 1995, 151, 77.

131 A. Charvat, A. Bögehold, A. Beinsen and B. Abel, Anal. Chem., 2007 , in press.

\section{Join the RSC and advance the chemical sciences}

RSC membership offers you the chance to play your part in promoting the chemical sciences.

Our 43,000 members come from diverse areas of the chemical sciences worldwide and enjoy:

- Unrivalled access to up to date scientific information

Exclusive discounts on books, journals and conferences

Vital career support and professional advice

Essential networking opportunities with over 43,000 members worldwide

Chemistry World and RSC News every month 